Published in Journal of Sound and Vibration 449, 98-120, 2019. Accepted 28/02/19.

DOI: 10.1016/j.jsv.2019.02.036

\title{
Application of a wavenumber domain numerical method to the prediction of the radiation efficiency and sound transmission of complex extruded panels
}

\author{
Hyungjun Kim ${ }^{\text {a }}$, Jungsoo Ryue ${ }^{\mathrm{a}, *}$, David J. Thompson ${ }^{\mathrm{b}}$ \\ and Angela D. Müller ${ }^{b}$
}

${ }^{a}$ School of Naval Architecture and Ocean Engineering, University of Ulsan, Ulsan, Korea
${ }^{\mathrm{b}}$ Institute of Sound and Vibration Research, University of Southampton, Southampton, UK

* : corresponding author: jsryue@ulsan.ac.kr

\begin{abstract}
Complex-shaped aluminium panels are adopted in many structures to make them lighter and stronger. The vibro-acoustic behaviour of these complex panels has been of interest for many years but conventional finite element and boundary element methods are not efficient in predicting their performance at higher frequencies. Where the cross-sectional properties of the panels are constant in one direction, which is the case for extruded panels, wavenumber domain numerical analysis can be applied and this becomes particularly suitable for panels with complex cross-sectional geometries. Because they are based on a twodimensional model, these methods can reduce the computational cost compared with other numerical methods using full three-dimensional models, while nevertheless including threedimensional effects. In this paper, a coupled waveguide finite element and boundary element method is applied to predict the radiation efficiency and sound transmission of a doublelayered aluminium extruded panel from a train carriage floor. The results are interpreted in the wavenumber domain from which the contributions of different types of waves can be identified. In the calculations, the air cavities between top and bottom panels are considered to examine their contributions to the vibro-acoustic behaviour of the panel. The predicted results are compared with measured ones obtained using a finite length panel. To reflect the
\end{abstract}


finite length of the actual panel used in the measurement, spatial window functions are applied to the sound transmission through the infinitely long panel, giving improved agreement with the measurements.

Keywords: aluminium extruded panel; radiation efficiency; sound transmission loss; waveguide finite element and boundary element method

\section{Introduction}

Complex-shaped stiffened panels are widely used nowadays in many structures such as railway vehicles, ships and offshore structures, in order to make them lighter and stronger. To predict the acoustic performance of these structures, it is essential to understand the vibroacoustic behaviour of such complex-shaped panels. As an example of such panels, an extruded aluminium panel used in the floor of a railway vehicle, which has a uniform crosssection along its length, is considered in this study.

For structures with uniform cross-sections like these extruded panels, wavenumber domain analysis becomes particularly suitable for predicting their vibro-acoustic performance. The radiation efficiency and sound transmission loss (STL) are two important vibro-acoustic properties that are investigated in this study. The radiation efficiency is a measure of sound radiation from a vibrating structure, defined as the radiated sound power normalised by the surface averaged mean-squared velocity, surface area and characteristic acoustic impedance of air. The STL is a measure of airborne sound transmission through a partition, given in decibels and defined as the incident sound intensity level minus the transmitted intensity level.

These properties have been studied previously for extruded panels by a few researchers. The radiation efficiency for a simple plate strip has been studied theoretically by Xie et al. [1] and they proposed simplified formulae which were used in modelling aluminium extrusions from railway vehicles [2]. A numerical analysis for the radiation efficiency of plate strips and sound transmission through them has been carried out by Prasetiyo [3] using a wavenumber domain numerical method. He found that the numerical results coincided well with analytical results.

For plate strips with longitudinal stiffeners, Orrenius and Finnveden [4] have examined the contributions of the stiffeners in terms of dispersion relations by using the waveguide finite element (WFE) method. This, also known as the 2.5D FE method, has 
previously been used for the vibration analysis of waveguide structures such as car tyres [5], rails $[6,7]$, plates [3], etc. From their study, it was found that at high frequencies waves travel mainly along the strips (or bays) which are bounded by two adjacent stiffeners. Kim and Ryue [8] have predicted the radiation efficiency for single and double layered stiffened plate strips numerically and investigated the effect on the results of the stiffeners and an added second layer. However, they only studied an extruded panel possessing a relatively simple cross-sectional geometry and did not investigate the STL. Zhang et al. [9] recently investigated the effect of different reinforcement rib arrangements on the STL through a complex shaped panel. Kim and Ryue [10] have examined the STL of an extruded panel used for a railway vehicle. They investigated the effects of where the panel is truncated in the periodic arrangement of stiffeners and found that the main influence appears below the first cut-on frequency of the global bending waves due to differences in stiffness of the strips at the edges.

In predicting the internal noise of a train constructed from extruded panels, Shaw [11] applied the statistical energy analysis (SEA) technique. This technique is useful for mid to high frequencies but it is not straightforward to apply it to extruded panels. Xie et al. [12] studied the mode count and modal density of extruded panels in an attempt to develop an SEA model. They used this model to predict the radiation efficiency of the extruded panel [2].

Chronopoulos et al. [13] used SEA combined with the 2D wave finite element method, also known as the periodic unit cell (PUC) method, to predict the radiation efficiency and STL for a sandwich panel. Kohrs [14] used the PUC method to investigate wave propagation through light-weight double-layered plates connected by inclined or vertical interior stiffeners. Orrenius et al. [15] reviewed both the PUC and 2.5D WFE methods and compared them for application to several structures including a complex-shaped extruded panel. In both methods, one-way coupling between the structure and outer fluid domains was assumed, making use of the Rayleigh integral. These studies have shown that these methods are applicable for predicting the radiation efficiency and STL of extruded panels.

The wavenumber domain analysis is based on the assumption that panels have infinite extent in the axial direction. To reflect the finite length of practical panels, Orrenius et al. [15] introduced a Rayleigh-Ritz procedure to the WFE method. However, the modal summation used in this combined method requires a high computational cost. Alternatively, a spatial window technique can also be applied to the wavenumber domain analysis. Villot et al. [16] applied a spatial window function in the width and length directions to results from an 
infinite panel to predict the radiation efficiency and STL through a simple panel. For the STL through orthogonally stiffened panels, Legault et al. [17] found that the spatial window technique is effective for the stiffened plates at mid to high frequencies where the bending wavelength of the panel is smaller than the stiffener spacing.

In terms of experimental study, Müller [18] has measured the radiation efficiency and sound transmission of an extruded panel with a complex geometry, which is a typical floor panel of a railway carriage. Nilsson et al. [19] have also measured the STL for the same extruded panel in a different test condition. In these experiments, Müller mounted the extruded panel in an aperture between two reverberation chambers, whereas Nilsson et al. used the reverberation and anechoic chambers. Orrenius et al. [15] also presented experimental results obtained using intensity scanning and compared the measured transmission losses for a train floor panel with those obtained from both the PUC and WFE methods.

In this paper, the radiation efficiency and STL of an extruded panel from a railway vehicle are investigated using the waveguide finite element and boundary element (WFE/BE) method. The WFE/BE approach has previously been used to predict the sound radiated from rails [20, 21], simple plate strips [3] and stiffened double plates [5, 9, 10]. In this work, the dependence of the radiation efficiency on two different types of excitation positions is investigated; a point force in the middle of the strip or on an interior stiffener. Also, the air cavities between the top and bottom panels are included to examine their contributions to the radiation efficiency and STL of the panel. To give insight into the radiation and transmission behaviours, results will be presented in the wavenumber domain, from which the contributions of different types of waves can be identified. In addition, to consider the finite length of the extruded panel in the prediction of the STL, a windowing technique $[16,17]$ is applied in this paper in the length direction. Finally, the numerical results are validated through comparison with experiments carried out with a finite length extruded panel.

\section{Waveguide finite element and boundary element analysis for an extruded panel}

In this section, the waveguide finite element and boundary element (WFE/BE) method is described briefly. This method is known to be particularly useful for waveguide structures which have a uniform cross-section along one direction ( $x$ direction in this paper), such as rails, plates, pipes, ducts, etc. Details of the derivation of the governing equations are well- 
reported in the literature $[5,20]$ so only a brief introduction of the method is given here. For the results presented in this paper, a software program, called WANDS [22], is used to

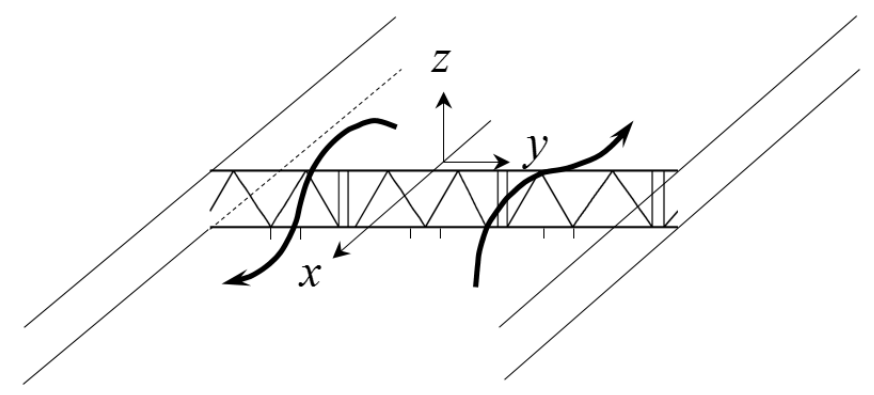

Fig. 1. Coordinates and scheme of wave propagation used in WFE analysis.

analyse the vibro-acoustic behaviour of the extruded panel.

In this wavenumber domain method, only a two-dimensional cross-section of the structure is meshed, assuming harmonic dependence on time and on the third direction in space, as indicated in Fig. 1. The governing equation of the WFEs for a waveguide structure modelled with plate elements is given by

$$
\left[\mathbf{K}_{s}(\kappa)-\omega^{2} \mathbf{M}_{s}\right] \tilde{\mathbf{\Phi}}=\tilde{\mathbf{F}}_{s},
$$

where $\tilde{\boldsymbol{\Phi}}$ is the nodal displacement vector in the wavenumber domain, $\omega$ is the angular frequency and $\kappa$ is the structural wavenumber along the $x$ direction and

$$
\mathbf{K}_{s}(\kappa)=\left(\mathbf{K}_{s 4}(-\mathrm{i} \kappa)^{4}+\mathbf{K}_{s 2}(-\mathrm{i} \kappa)^{2}+\mathbf{K}_{s 1}(-\mathrm{i} \kappa)+\mathbf{K}_{s 0}\right)(1+\mathrm{i} \eta)
$$

in which $\mathbf{K}_{s 4}, \mathbf{K}_{s 2}, \mathbf{K}_{s 1}$, and $\mathbf{K}_{s 0}$ are related to the stiffness of the structure and $\eta$ is the damping loss factor (which may be frequency-dependent). $\mathbf{M}_{s}$ is the mass matrix and $\tilde{\mathbf{F}}_{s}$ is the vector of excitation forces at these nodes. For free vibration, i.e. $\tilde{\mathbf{F}}_{s}=\mathbf{0}$ and $\eta=0$, Eq. (2) can be solved for $\omega$ at given real wavenumbers. This general eigenvalue problem provides dispersion relations of waves propagating along the $x$ direction of the structure.

If the internal region of the structure is filled with a fluid, such as air, this can also be modelled with WFEs. The WFE equation for the fluid is given by

$$
\left[\mathbf{K}_{f}(\kappa)-\omega^{2} \mathbf{M}_{f}\right] \tilde{\mathbf{\Psi}}_{\mathrm{in}}=\tilde{\mathbf{F}}_{f}
$$

where

$$
\mathbf{K}_{f}(\kappa)=\mathbf{K}_{f 2}(-\mathrm{i} \kappa)^{2}+\mathbf{K}_{f 0}
$$


in which $\mathbf{K}_{f 2}$ and $\mathbf{K}_{f 0}$ are the 'mass' matrices of the internal fluid and $\mathbf{M}_{f}$ is its 'stiffness' matrix, which are derived from the kinetic and potential energies, respectively [22]. $\tilde{\boldsymbol{\Psi}}_{\text {in }}$ is the velocity potential at the WFE nodes of the internal fluid and $\tilde{\mathbf{F}}_{f}$ is the vector of external forces that excite the fluid. The potential formulation is used in preference to a displacement formulation as it requires only a single degree of freedom at each node point and also the velocity potential can be coupled to the structure as a damping. Accounting for the coupling condition between the internal fluid and the structure, the combined governing equation becomes

$$
\left[\left[\begin{array}{cc}
\mathbf{K}_{s}(\kappa) & \mathbf{0} \\
\mathbf{0} & \mathbf{K}_{f}(\kappa)
\end{array}\right]+\mathrm{i} \omega\left[\begin{array}{cc}
\mathbf{0} & \mathbf{C}_{3} \\
-\mathbf{C}_{3}^{\mathbf{T}} & \mathbf{0}
\end{array}\right]-\omega^{2}\left[\begin{array}{cc}
\mathbf{M}_{s} & \mathbf{0} \\
\mathbf{0} & \mathbf{M}_{f}
\end{array}\right]\right]\left\{\begin{array}{c}
\tilde{\mathbf{\Phi}} \\
\tilde{\boldsymbol{\Psi}}_{\mathrm{in}}
\end{array}\right\}=\left\{\begin{array}{c}
\tilde{\mathbf{F}}_{s} \\
\tilde{\mathbf{F}}_{f}
\end{array}\right\},
$$

where $\mathbf{C}_{3}$ is the coupling matrix between the waveguide structure and internal fluid and the superscript $\mathrm{T}$ denotes a matrix transpose. The dispersion relations of this coupled system can be obtained by converting Eq. (5) into a generalized eigenvalue problem by setting the righthand side to zero [23].

When the exterior of the waveguide structure is connected to a fluid domain, wavenumber domain boundary elements (WBE) are introduced on the periphery of the structure to model the external fluid and these are coupled with the WFEs. The sound pressure and the fluid particle velocity in the direction normal to the fluid boundary are expressed as

$$
\tilde{p}=\mathrm{i} \omega \rho_{0} \tilde{\Psi}_{\mathrm{ex}}, \quad \tilde{v}_{n}=-\frac{\partial \tilde{\Psi}_{\mathrm{ex}}}{\partial \mathbf{n}},
$$

where $\tilde{\Psi}_{\mathrm{ex}}$ and $\partial \tilde{\Psi}_{\mathrm{ex}} / \partial \mathbf{n}$ are the velocity potential and the normal velocity at the nodes of the boundary elements and $\mathbf{n}$ denotes the unit normal directional vector pointing out of the acoustic domain. For a given frequency $\omega$ and axial wavenumber $\kappa$, the governing equation for the external fluid is given by

$$
\mathbf{H}(\kappa) \tilde{\boldsymbol{\Psi}}_{\mathrm{ex}}-\mathbf{G}(\kappa) \frac{\partial \tilde{\boldsymbol{\Psi}}_{\mathrm{ex}}}{\partial \mathbf{n}}=\frac{\tilde{\mathbf{P}}_{i}}{\mathrm{i} \omega}
$$

where $\tilde{\mathbf{P}}_{i}$ are the incident acoustic pressures acting at the nodes of WBEs, $\mathbf{H}(\kappa)$ and $\mathbf{G}(\kappa)$ are matrices of acoustic Green's functions for wavenumber $\kappa$. In the coupling between the WFEs and the external fluid, the pressure from the external fluid is applied as an 
additional load to the structure. Then the equation of the structure coupled with the external fluid is given by

$$
\left[\mathbf{K}_{s}(\kappa)-\omega^{2} \mathbf{M}_{s}\right] \tilde{\mathbf{\Phi}}=\tilde{\mathbf{F}}_{s}+\mathrm{i} \omega \rho_{0} \mathbf{C}_{1} \tilde{\mathbf{\Psi}}_{\mathrm{ex}},
$$

where $\mathbf{C}_{1}$ denotes the coupling matrix between the WFEs and WBEs and $\rho_{0}$ is the fluid density. In addition, the continuity condition of the normal velocity on the 'wet' surface is given by

$$
\mathbf{I} \frac{\partial \tilde{\mathbf{\Psi}}_{\mathrm{ex}}}{\partial \mathbf{n}}-\mathrm{i} \omega \mathbf{C}_{2} \tilde{\mathbf{\Phi}}=\mathbf{0}
$$

where $\mathbf{I}$ is the identity matrix and $\mathbf{C}_{2}$ is the matrix transforming WFE displacements onto the normal directions of the coupled WBEs. For the coupling between two-noded BEs having a linear shape function and two-noded plate FEs having a cubic shape function, $\mathbf{C}_{2}$ can be determined as

$$
\left[\begin{array}{c}
\tilde{v}_{n, 1} \\
\tilde{v}_{n, 2}
\end{array}\right]=\mathrm{i} \omega\left[\begin{array}{cccc}
1 & 0 & 0 & 0 \\
0 & 0 & 1 & 0
\end{array}\right] \mathbf{T}\left[\begin{array}{c}
\tilde{v}_{1} \\
\tilde{w}_{1} \\
\tilde{\theta}_{1} \\
\tilde{v}_{2} \\
\tilde{w}_{2} \\
\tilde{\theta}_{2}
\end{array}\right],
$$

where $\tilde{v}_{n, j}$ is the normal velocity at $j$ th node of each WBE, $\mathbf{T}$ is the transformation matrix from the global coordinates to the local coordinates and is given in Eq. (11.29) in ref. [22]. $\tilde{v}_{j}$ and $\tilde{w}_{j}$ are the displacements in the $y$ and $z$ directions and $\tilde{\theta}_{j}$ is the rotational angle in the $x$ direction at $j$ th node of each WFE. For WBEs not coupled with WFEs, constraints are needed for the uncoupled WBE nodes. The boundary conditions are written in matrix form as

$$
\mathrm{i} \omega \rho_{0} \mathbf{C}_{a} \tilde{\boldsymbol{\Psi}}_{\mathrm{ex}}-\mathbf{C}_{b} \frac{\partial \tilde{\boldsymbol{\Psi}}_{\mathrm{ex}}}{\partial \mathbf{n}}=\mathbf{C}_{c} .
$$

For example, fixed boundary conditions are specified by $C_{a}=0, C_{b}=1$ and $C_{c}=0$ at each node on the boundary. For the coupled analysis of the complete system, the governing equation of the WFE/BEs is given by 


$$
\left[\begin{array}{cccc}
\mathbf{K}_{s}(\kappa)-\omega^{2} \mathbf{M}_{s} & \mathrm{i} \omega \mathbf{C}_{3} & -\mathrm{i} \omega \rho_{0} \mathbf{C}_{1} & \mathbf{0} \\
-\mathrm{i} \omega \mathbf{C}_{3}^{\mathbf{T}} & \mathbf{K}_{f}(\kappa)-\omega^{2} \mathbf{M}_{f} & \mathbf{0} & \mathbf{0} \\
\mathbf{0} & \mathbf{0} & \mathbf{H}(\kappa) & -\mathbf{G}(\kappa) \\
-\mathrm{i} \omega \mathbf{C}_{2} & \mathbf{0} & \mathbf{0} & \mathbf{I} \\
\mathbf{0} & \mathbf{0} & \mathrm{i} \omega \rho_{0} \mathbf{C}_{a} & -\mathbf{C}_{b}
\end{array}\right]\left\{\begin{array}{c}
\tilde{\boldsymbol{\Phi}} \\
\tilde{\boldsymbol{\Psi}}_{\text {in }} \\
\tilde{\boldsymbol{\Psi}}_{\text {ex }} \\
\partial \tilde{\boldsymbol{\Psi}}_{\mathrm{ex}} \\
\hline \partial \mathbf{n}
\end{array}\right\}=\left\{\begin{array}{c}
\tilde{\mathbf{F}}_{s} \\
\tilde{\mathbf{F}}_{f} \\
\tilde{\mathbf{P}}_{i} / \mathrm{i} \omega \\
\mathbf{0} \\
\mathbf{C}_{c}
\end{array}\right\}
$$

Once $\tilde{\boldsymbol{\Psi}}_{\mathrm{ex}}$ and $\partial \tilde{\boldsymbol{\Psi}}_{\mathrm{ex}} / \partial \mathbf{n}$ have been found from Eq. (12), the sound pressure and fluid particle velocity at the fluid nodes are obtained in the wavenumber domain from Eq. (6).

\section{Modelling the panel and fluid}

In this study, the extruded panel shown in Fig. 2(a), which is a part of typical floor panel of a railway vehicle, is examined by WFE/BE analysis. The panel is made of aluminium but the top plate is covered with a heavy rubber mat. The cross-sectional model of the panel structure is illustrated in Fig. 2(b); this is composed of two-noded plate elements with a cubic shape function. The rubber mat attached on the top plate is included in the model by increasing the mass and damping of the top plate. The stiffening effects by the rubber mat is excluded in this modelling because the Young's modulus of the rubber is much lower than that of the aluminium. In principle, it would be possible to introduce an additional WFE layer

(a)

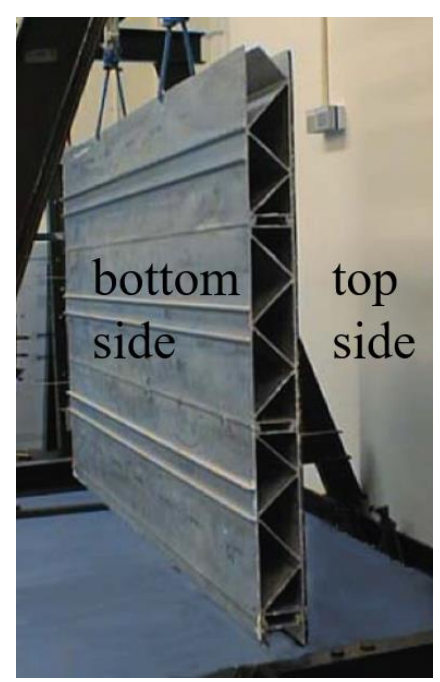

(b)

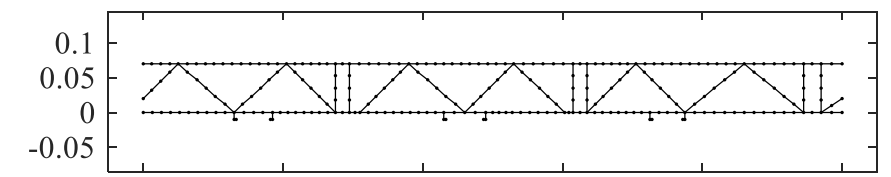

(c)

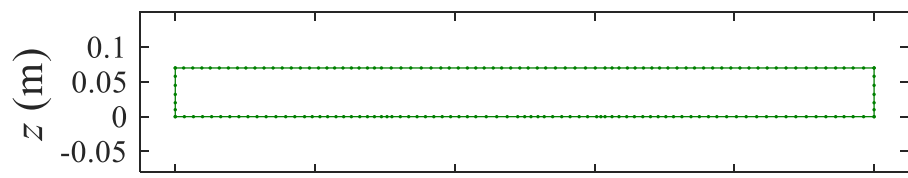

(d)

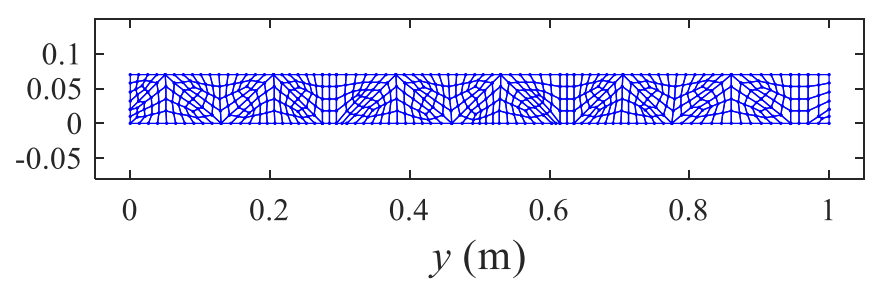

Fig. 2. (a) A specimen of an extruded floor panel for a railway vehicle. (b) Cross-sectional WFE model of the extruded panel, (c) WBE model for external air and (d) WFE model for internal air. (Dots represent nodes in each model.) 
Table 1. Properties and dimensions of the extruded panel.

\begin{tabular}{cccccc}
\hline Material & Model & $\begin{array}{c}\text { Thickness } \\
(\mathrm{mm})\end{array}$ & $\begin{array}{c}\text { Density } \\
\left(\mathrm{kg} / \mathrm{m}^{3}\right)\end{array}$ & $\begin{array}{c}\text { Poisson's } \\
\text { ratio }\end{array}$ & $\begin{array}{c}\text { Young's } \\
\text { modulus (GPa) }\end{array}$ \\
\hline \multirow{4}{*}{ Aluminium } & Top plate & 2.8 & & & \\
& Bottom plate & 2.7 & 2700 & 0.3 & 70 \\
& Vertical stiffeners & 2.3 & & & - \\
\hline Rubber & Oblique stiffeners & 2.6 & & & \\
\hline
\end{tabular}

for the rubber mat but this would require the use of solid elements rather than plates and would increase the mesh size considerably. Each plate element has an average length of about $12.5 \mathrm{~mm}$ and it was checked that this element size is acceptable to represent wave modes up to $5 \mathrm{kHz}$. (The model has about five nodes per bending wavelength along the $y$ direction in each bay for the highest order waves at $5 \mathrm{kHz}$.) The dimensions and properties of the panel are listed in Table 1. To consider the stiffening effects of the joints where the interior stiffeners are connected to top or bottom plate, the WFEs close to the joints are modelled with a thickness that is $20 \%$ larger than the remaining plates.

The damping loss factors of the top and bottom plates of the panel were measured in experiments by Zhang et al. [9]. The panel was freely suspended and the damping loss factors for each bay of the top and bottom plates are obtained using the energy method [24]. The damping loss factors for each bay can be yielded as [9]

$$
\eta_{i}=\frac{\operatorname{Re}\left\{Y_{p}\right\}}{m \omega\left\langle\left|Y_{t}\right|^{2}\right\rangle},
$$

where $\eta_{i}$ is the damping loss factor measured at the $i$ th bay, $Y_{p}$ is the point mobility, $m$ is the mass of the extruded panel, $Y_{t}$ is the transfer mobility, and $\langle\cdot\rangle$ denotes the spatially averaged quantity. The transfer mobilities were measured in each bay with the fixed accelerometer varying the impact position along the bay. In total, 50 points were randomly excited to measure the spatially-averaged mean squared transfer mobility. The measured point mobilities in each bay are presented in Sec. 5.1.

In the present modelling, the two damping loss factors for the top and bottom plates are evaluated by means of the reciprocal average of the values measured in each bay, given by 


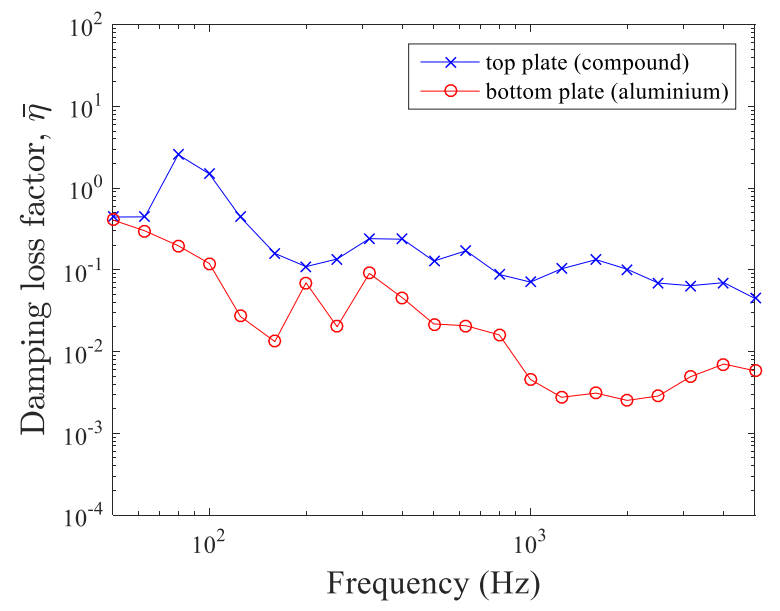

Fig. 3. The damping loss factors for the top compound plate and the bottom purely aluminium plate.

$$
\bar{\eta}=\frac{N}{\sum_{i=1}^{N} \frac{1}{\eta_{i}}}
$$

where $\bar{\eta}$ denotes the averaged damping loss factor and $N$ is the number of bays measured in each top and bottom plate. This scheme of averaging is based on the fact that the low values of damping will dominate the response and hence the sound radiation and transmission.

The averaged damping loss factors for the top and bottom plates are illustrated in Fig. 3. This shows that damping of the top plate is greater than that of the bottom plate due to the rubber mat and they both decrease in general as frequency increases. These damping loss factors are used for the calculation. The interior stiffeners of the panel are assigned the same damping as the bottom plate.

In the WFE modelling for the radiation efficiency, the panel is set to have free-free boundary conditions at both ends for compatibility with the measurements described in Sec. 5 below. The acoustic model needs to have a closed boundary because a direct BE method is implemented for the WBEs. Due to the complicated layout of the interior stiffeners, a proper coupling between the WFEs and WBEs at both ends would be quite challenging. So, for simplicity, the WBEs enclose the panel in a rectangular shape, as shown in Fig. 2(c). Since the height of the panel (about $0.07 \mathrm{~m}$ ) is relatively small compared with the width of the panel $(1 \mathrm{~m})$, it is expected that most of the sound will be radiated from the top and bottom plates. Hence, the WBEs on both sides are assigned to have zero velocity. The total number 
of dofs in the model in Fig. 2(b) is 1364, consisting of 1012 for the WFEs (268 elements) and 352 for the WBEs (172 elements).

For the case of sound transmission, two separate WBE domains are located on the top and bottom plates (at $z=0$ and $z=0.07 \mathrm{~m}$ in Fig. 2(c)) and it is assumed that each panel is rigidly baffled for $y \leq 0$ and $y \geq 1 \mathrm{~m}$ by using half-space Green's functions in the WBE formulation. In the experiments discussed in Sec. 5, the sound transmission was measured by installing the panel in a frame set in a wall aperture between two rooms. The practical boundary conditions of the panel were somewhere between simply supported and fixed. In the numerical study, these two boundary conditions are applied at both ends of the panel to examine their effect on the sound transmission of the panel. For the simply supported boundary condition, the number of total dofs is 1324 , which consists of 1000 for the WFEs (268 elements) and 324 for the WBEs (160 elements). For the fixed condition, the total number of dofs is 1320, of which 996 are for the WFEs.

The internal air modelled with WFEs in Fig. 2(d) is composed of 519 four-noded quadrilateral elements with linear shape functions. This fluid domain has 721 dofs.

\section{Numerical results for the extruded panel}

\subsection{Dispersion relation}

In order to understand the characteristics of waves propagating along the extruded panels with different boundary conditions, dispersion diagrams are illustrated in Fig. 4, obtained from WFE models of the panels (i.e. without coupling with the interior or exterior fluid). These are based on 201 logarithmically spaced wavenumbers from $10^{-1}$ to $10^{2}$ $\mathrm{rad} / \mathrm{m}$ with the corresponding frequencies identified from the eigenvalue analysis of Eq. (2). For comparison, the acoustic wavenumber is also plotted with a dashed line.

It can be seen from Fig. 4 that the effects of the boundary conditions are more obvious in the low frequency range. There are several waves at low frequencies in the case of the freefree boundary condition, as shown in Fig. 4(a). These correspond to global motions of the extruded panel, i.e. the vertical bending, torsion, lateral bending and longitudinal waves of the whole section, in descending order of the wavenumber at $100 \mathrm{~Hz}$. On the other hand, the panel with the simply supported boundary condition (Fig. 4(b)) does not have any wave below about $87 \mathrm{~Hz}$. However, at frequencies above about $400 \mathrm{~Hz}$ and at high wavenumbers, 
many local modes occur in the plate strips and the dispersion diagrams in Figs 4(a) and 4(b) show similar features.

For the extruded panel with the free-free boundary condition in Fig. 4(a), an equivalent single plate can be introduced, which possesses the same dispersion relation as the first vertical bending wave in the extruded panel. The density of this equivalent plate is obtained by using the longitudinal wave speed, $c_{l}=\sqrt{E / \rho_{e}}$ where $E$ is Young's modulus of aluminium and $\rho_{e}$ is the density of the equivalent panel. Since $c_{l}$ evaluated from the longitudinal wave in Fig. 4(a) is $4584 \mathrm{~m} / \mathrm{s}, \rho_{e}$ becomes $3380 \mathrm{~kg} / \mathrm{m}^{3}$. The thickness, $h_{e}$, of the equivalent plate is specified by the radius gyration, $r_{e}$, which is given by $r_{e}=h_{e} / \sqrt{12}=c_{b}^{2} / c_{l} \omega$ where $c_{b}$ is the bending wave speed. $c_{b}$ is evaluated from Fig. 4(a) as $206 \mathrm{~m} / \mathrm{s}$ at $50 \mathrm{~Hz}$. So, $h_{e}$ is found to be $10 \mathrm{~cm}$. The vertical bending wavenumber of this equivalent plate is plotted in Fig. 4 with the dash-dotted line. Since this bending wave has global vertical deformation, its bending stiffness is predominantly governed by the interior stiffeners, particularly by the six vertical stiffeners, located between the top and bottom plates.

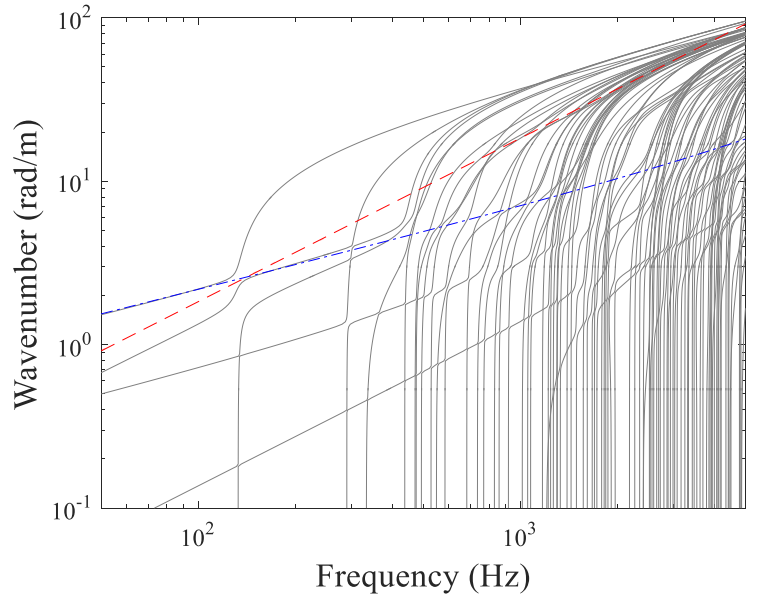

(a)

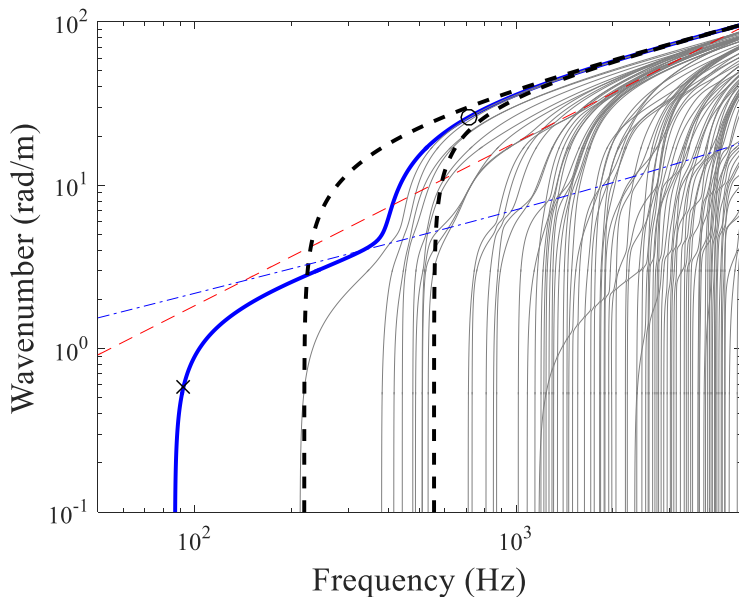

(b)

Fig. 4. Dispersion diagrams of the extruded panel with (a) free boundary conditions and (b) simply supported boundary conditions. (Acoustic wavenumber is added with a thin dashed line; the vertical bending wave of an equivalent plate is presented with a dashdotted line; two thick dashed lines in Fig. 4(b) are two first order waves of a single bay of the top plate having a width of $0.153 \mathrm{~m}$ with simply supported and fixed boundary conditions at both ends.) 


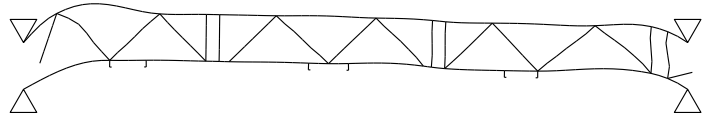

(a)

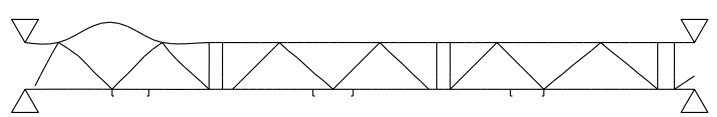

(b)

Fig. 5. The deformation shapes of two waves marked in Fig. 4(b) with (a) ' $x$ ' at $91 \mathrm{~Hz}$ and $0.6 \mathrm{rad} / \mathrm{m}$, and (b) ' $\bigcirc$ ' at $710 \mathrm{~Hz}$ and $26 \mathrm{rad} / \mathrm{m}$.

It is seen from Fig. 4 that most of the waves change their dispersion characteristics as frequency and wavenumber increase. To observe these features, cross-sectional deformation shapes of the first wave (the thick solid line in Fig. 4(b)) at two different frequencies (and wavenumbers) marked with ' $x$ ' and ' $O$ ' are shown in Fig. 5. It can be seen from Fig. 5(a) that the wave possesses a global deformation of the panel at $91 \mathrm{~Hz}$ and $0.6 \mathrm{rad} / \mathrm{m}$, marked with ' $x$ '. On the contrary, Fig. 5(b) shows that this wave is converted to a wave localized in a strip (or bay), between two adjacent inclined interior stiffeners at $710 \mathrm{~Hz}$ and $26 \mathrm{rad} / \mathrm{m}$, marked with ' $\bigcirc$ '. Fig. 5(a) also shows that the left-end $(y=0)$ of the panel is much flexible compared to the other end at $y=1 \mathrm{~m}$ due to the disconnection with the interior stiffener.

In order to see the asymptotic behaviour of the local waves occurring in the top plate, the waves propagating along a single bay with a width of $0.153 \mathrm{~m}$ (which is typical of the strips in the top panel) were investigated for two different ideal boundary conditions: simply supported or fixed [4]. The two cut-on frequencies are $217 \mathrm{~Hz}$ for simply supported boundary condition and $553 \mathrm{~Hz}$ for fixed boundary condition. These two first-order mode waves in the strip for each boundary condition are illustrated in Fig. 4(b) with thick dashed lines. It can be observed that three waves, including the one in thick solid line, are bounded by these two asymptote waves of a single bay. (These three are the waves localized in three wide bays in the top plate, respectively.) It can be deduced from this that the extruded panel begins to have localized deformation within a bay from around $400 \mathrm{~Hz}$. In ref. [18], it was also found from experiments that vibration modes localized within a bay in the top plate exist above $400 \mathrm{~Hz}$.

In addition, it is noted that all the dispersion curves in Fig. 4(a) or (b) do not cross each other but instead the waves swap their wave modes when two dispersion curves come close [25]. In order to see the conversion of the wave modes, the dispersion diagram in Fig. 4(b) is replotted in linear frequency-wavenumber scale in Fig. 6(a) and a single wave curve is chosen. The wave curve chosen is distinguished with a thick solid line in Fig. 6(a) and six frequency-wavenumber points selected are marked with symbols. To display the variation of 


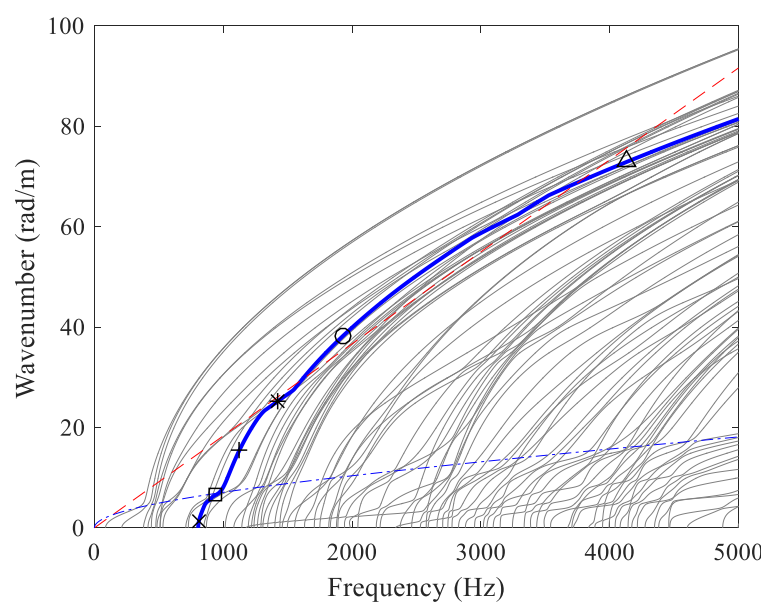

(a)

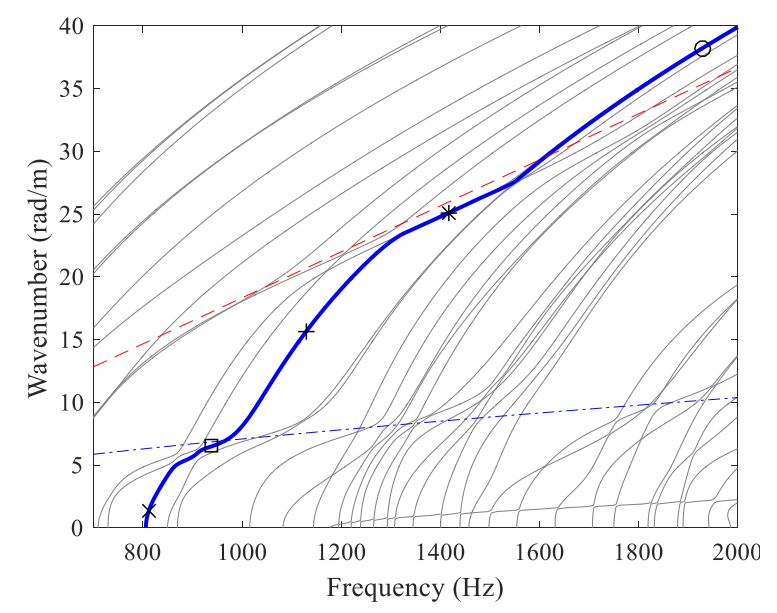

(b)

Fig. 6. (a) The dispersion diagram of the extruded panel in Fig. 4(b) replotted in linear scale and (b) the magnified diagram between $700 \mathrm{~Hz}$ and $2 \mathrm{kHz}$. (The thin dashed and dashdotted lines are the same as in Fig. 4(b).)

the wave modes in the chosen curve more clearly, a magnified diagram of Fig. 6(a) is plotted in Fig. 6(b).

The cross-sectional deformations of the six waves marked are shown in Fig. 7. Fig. 7(a) shows that the wave chosen cuts on with a global deformation of the panel. Then, this wave in Fig. 7(b) is converted to a wave which is governed by the vertical bending stiffness of the internal stiffeners so that the slope of the wave curve is largely declined (i.e., the bending stiffness is increased) [4]. Since the six vertical interior stiffeners are very stiff in the $z$ direction, their vertical deformations are not much visible in Fig. 7(b) although they govern the global panel behaviour. Then, at $1129 \mathrm{~Hz}$ and $15.6 \mathrm{rad} / \mathrm{m}$ (marked with ' + '), the

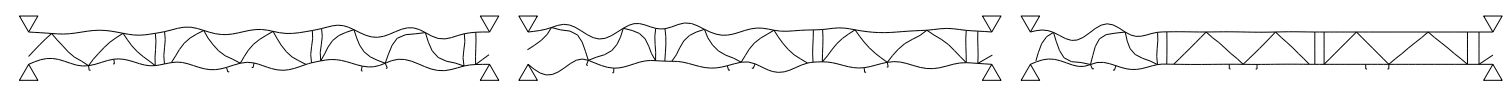

(a)

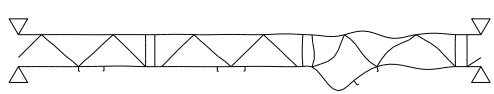

(d) (b)

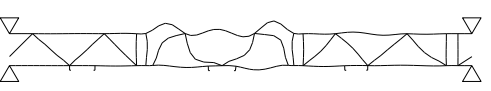

(e) (c)

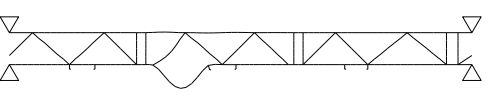

(f)

Fig. 7. The deformation shapes of the wave chosen in Fig. 6. (a) ' $x$ ' at $812 \mathrm{~Hz}$ and $1.4 \mathrm{rad} / \mathrm{m}$, (b) ' $\square$ ' at $939 \mathrm{~Hz}$ and $6.5 \mathrm{rad} / \mathrm{m}$, (c) '+' at $1129 \mathrm{~Hz}$ and $15.6 \mathrm{rad} / \mathrm{m}$, (d) ' *' at 1418 $\mathrm{Hz}$ and $25.1 \mathrm{rad} / \mathrm{m}$, (e) ' $\bigcirc$ ' at $1929 \mathrm{~Hz}$ and $38.2 \mathrm{rad} / \mathrm{m}$, and (f) ' $\triangle$ ' at $4135 \mathrm{~Hz}$ and $72.9 \mathrm{rad} / \mathrm{m}$. 
wave has a localized deformation restricted to the left one-third section of the panel which is bordered by the pair of vertical internal stiffeners as shown in Fig. 7(c). According to Fig. 6(b), there are two more wave curves in parallel on the lefthand side of the wave chosen and they also have localized deformations within the middle and right one-third sections of the panel, respectively. The deformation shape of the wave marked with ' $*$ ' is illustrated in Fig. 7(d). Fig. 7(d) shows that this wave is localized mainly within a single bay in the right onethird region of the bottom plate. This bay has an external stiffener in the middle which stiffens the bay so that the slope of the wave curve becomes declined as shown in Fig. 6(b). The wave type at $1929 \mathrm{~Hz}$ and $38.2 \mathrm{rad} / \mathrm{m}$ where marked with ' $\bigcirc$ ' is shown in Fig. 7(e). This wave now has large deformations of two narrow bays and inclined interior stiffeners in the mid one-third section of the top plate. At a higher frequency of $4135 \mathrm{~Hz}$ and $72.9 \mathrm{rad} / \mathrm{m}$ (marked with ' $\triangle$ '), the wave type is illustrated in Fig. 7(f). This wave is localized within a region bounded by interior and exterior stiffeners in the bottom plate which is narrower than the width of the bay.

The dispersion diagrams for the panel coupled with the internal fluid are shown in Fig. 8. It is seen from comparison with Fig. 8 that strong coupling between the structure and internal air occurs around the acoustic wavenumber. For the free-free boundary condition in Fig. 8(a), global wave modes of the panel interact strongly with the internal acoustic wave at low frequencies. On the other hand, for the simply supported boundary condition in Fig. 8(b), relatively weak interactions take place at low frequencies because the structural wavenumbers of the global wave modes are lower than the acoustic wavenumbers.

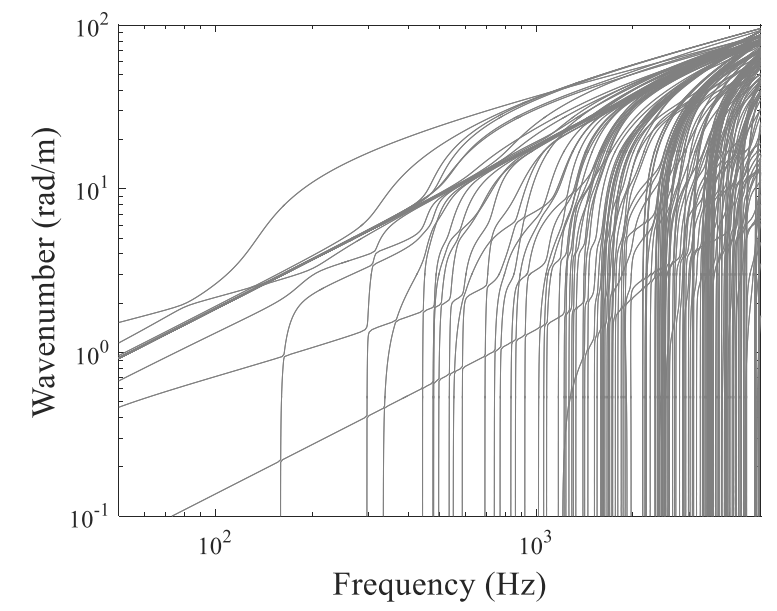

(a)

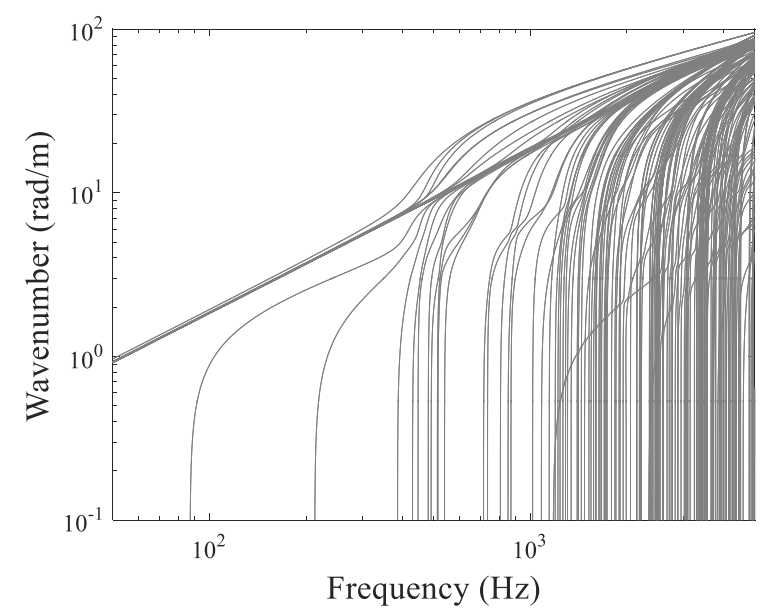

(b)

Fig. 8. Dispersion diagrams of the extruded panel coupled with the internal air with (a) free boundary conditions and (b) simply supported boundary conditions. 


\subsection{Radiation efficiency of the extruded panel}

\subsubsection{Calculation of the radiation efficiency}

The radiation efficiency $(\sigma)$ of the waveguide structure can be determined by

$$
\sigma=\frac{\Pi_{\mathrm{rad}}}{\rho_{0} c_{0} \Gamma\left\langle\overline{v^{2}}\right\rangle_{\mathrm{inf}}},
$$

where $c_{0}$ is the sound speed in the fluid, $\Gamma$ is the perimeter of the cross-section in contact with the fluid and $\left\langle\overline{v^{2}}\right\rangle_{\text {inf }}$ represents the integral of the mean-squared velocity over the length and averaged over the width. This differs from the usual definition of $\sigma$ due to the infinite extent of the panel in the waveguide direction. The radiated power, $\Pi_{\mathrm{rad}}$, and average mean-squared velocity, $\left\langle\overline{v^{2}}\right\rangle_{\text {inf }}$, of the panel in Eq. (15) can be obtained from

$$
\begin{gathered}
\Pi_{\mathrm{rad}}=\frac{1}{4 \pi} \operatorname{Re}\left\{\int_{-k}^{k} \int_{\Gamma} \tilde{p}(\kappa, y) \tilde{v}_{n}^{*}(\kappa, y) \mathrm{d} y \mathrm{~d} \kappa\right\}, \\
\left\langle\overline{v^{2}}\right\rangle_{\mathrm{inf}}=\frac{1}{2 \pi \Gamma} \int_{-\infty}^{\infty} \int_{\Gamma}\left|\frac{\tilde{v}_{n}(\kappa, y)}{2}\right|^{2} \mathrm{~d} y \mathrm{~d} \kappa,
\end{gathered}
$$

where $k=\omega / c_{0}$ is the acoustic wavenumber and the superscript $*$ denotes the complex conjugate. In Eq. (16), the integration with respect to $\kappa$ is limited to $\pm k$ because the panel does not radiate sound into the far field for $\kappa>k$. Since the sound pressure, $\tilde{p}(\kappa, y)$, and velocity, $\tilde{v}_{n}(\kappa, y)$, can be found from Eq. (6), Eqs (16) and (17) can be evaluated from the WFE/BE solutions without transforming the results from the wavenumber domain into the spatial domain.

\subsubsection{Predicted results for the radiation efficiency}

In the prediction of the radiation efficiency, a unit amplitude point force is applied at two different types of location on the bottom plate; one is in the middle of a strip between two interior stiffeners and the other is where an interior stiffener is connected to the plate. These positions correspond to P1 (or P2) on the strip and P3 and P4 on the stiffener as specified in Sec. 5.2.1 below. 
The radiation efficiency was calculated at 175 logarithmically spaced frequencies from $50 \mathrm{~Hz}$ to $5 \mathrm{kHz}$. It was validated that the result does not change significantly if the number of frequency steps is doubled. For the prediction of the radiated power, the wavenumbers at each frequency were set to have 512 evenly spaced points from $-k$ to $k$. It was confirmed that convergence of the results was achieved with this number of wavenumbers. The same number of wavenumbers was also used for the calculation of the averaged mean-squared velocity but the wavenumber span for the integral with respect to $\kappa$ in Eq. (17) was set to $100 \mathrm{rad} / \mathrm{m}$ to encompass the entire range of propagating waves at each frequency. The calculation time for the average mean-squared velocity and radiated sound power at each frequency is in each case about 50 seconds using a $3.5 \mathrm{GHz}$ interior computer with 8GB RAM. If the internal air cavity is added, the calculation time increases by about a factor of three.

For the panel model disregarding the internal air, the average mean-squared velocity and the sound power radiated from both the top and bottom plates are illustrated in Figs 9 and 10, as image plots against the frequency and wavenumber. The results in Fig. 9 are obtained for a point force applied in the middle of a strip at $y=0.528 \mathrm{~m}$ (P1 or P2), whereas the results in Fig. 10 correspond to a position on a stiffening rib at $y=0.635 \mathrm{~m}$ (P3). The acoustic wavenumber is also plotted with a dashed line. Additionally, the vertical bending wavenumber of the equivalent plate displayed in Fig. 4(a) is presented with a dash-dotted line in Figs 9 and 10.

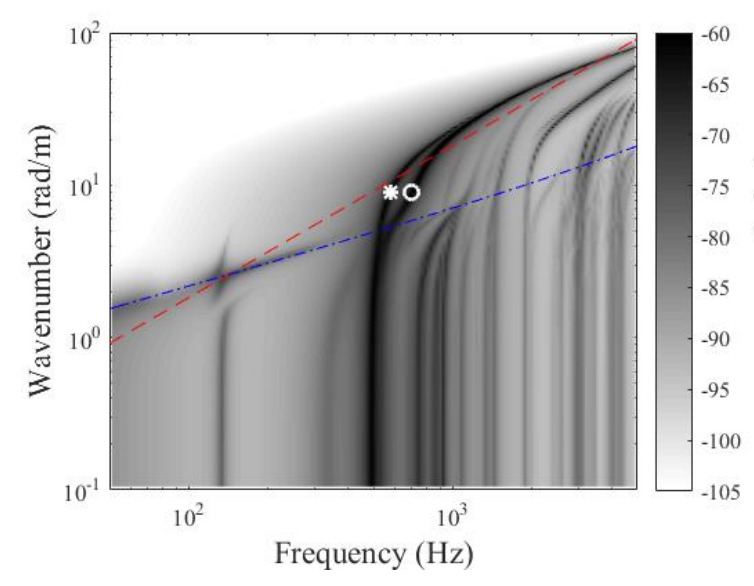

(a)

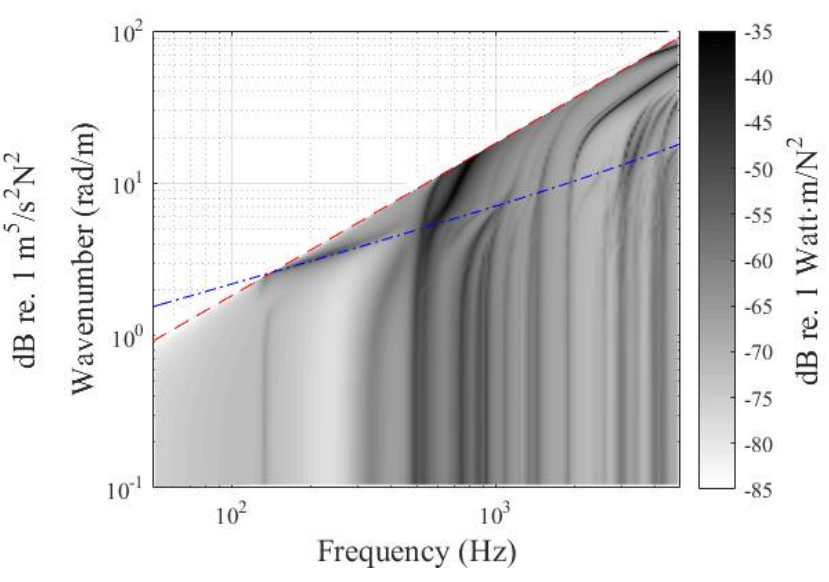

(b)

Fig. 9. Image plots of (a) the average mean-squared velocity and (b) radiated sound power of the panel for excitation in the middle of a strip at $y=0.528 \mathrm{~m}$, plotted against frequency and wavenumber. 


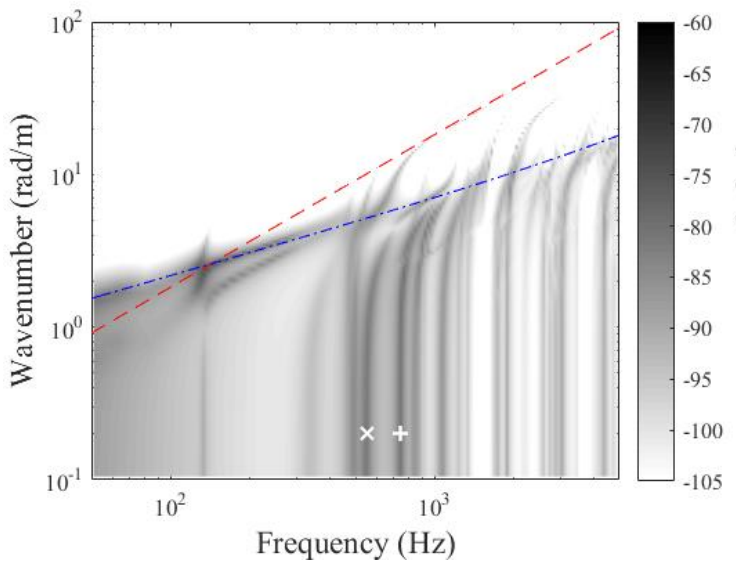

(a)

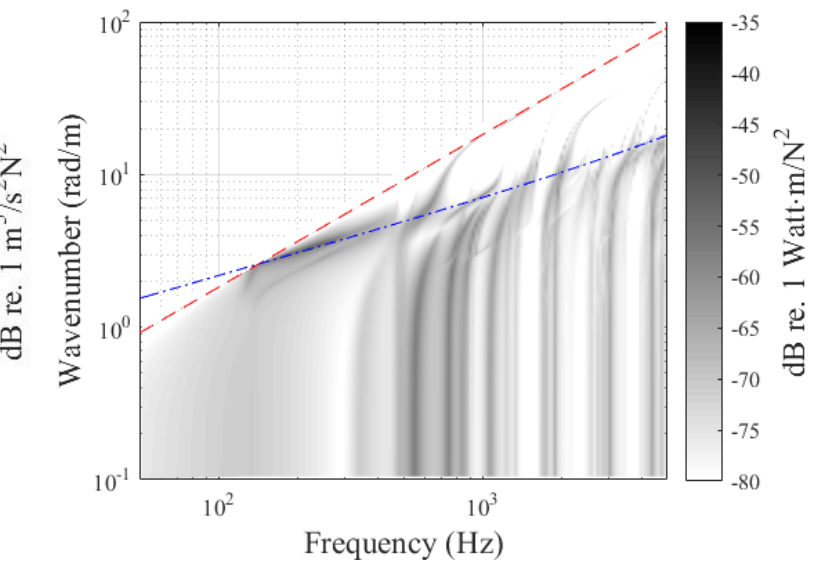

(b)

Fig. 10. Image plots of (a) the average mean-squared velocity and (b) radiated sound power of the panel for excitation on a stiffener at $y=0.635 \mathrm{~m}$, plotted against frequency and wavenumber.

For the excitation in the middle of the strip, as shown in Fig. 9, a small number of global wave modes generate the vibration at frequencies below about $400 \mathrm{~Hz}$ and many waves having higher order cross-sectional deformations contribute above about $400 \mathrm{~Hz}$. However, in terms of the radiated sound power, the waves which have wavenumbers larger than that of the acoustic wave do not contribute at all, see Fig. 9(b). Also, because of the higher damping of the top plate, strong vibration and radiation at high frequencies mainly come from the local waves in the bottom plate. It is seen from Fig. 9(a) that two wave curves possess strong vibration. The deformation shapes of them at $579 \mathrm{~Hz}$ ('*'), and $700 \mathrm{~Hz}$ (' $\bigcirc$ ')

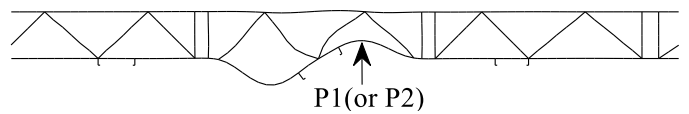

(a)

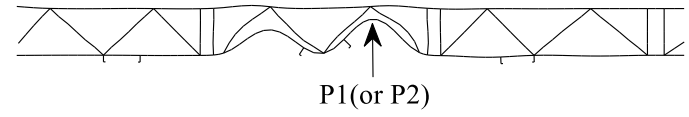

(b)

Fig. 11. The deformation shapes of the waves marked with (a) ' $*$ ' at $579 \mathrm{~Hz}$ and $8.9 \mathrm{rad} / \mathrm{m}$ and (b) ' $\bigcirc$ ' at $700 \mathrm{~Hz}$ and $8.9 \mathrm{rad} / \mathrm{m}$ in Fig. 9(a).

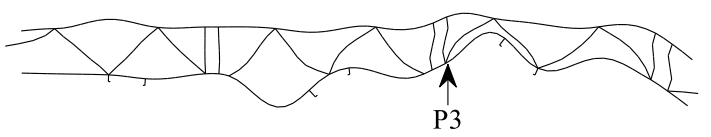

(a)

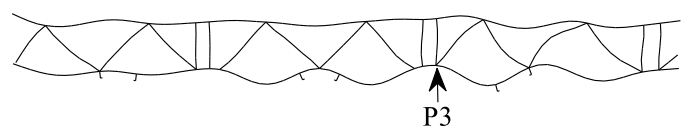

(b)

Fig. 12. The deformation shapes of the waves marked with (a) ' $x$ ' at $556 \mathrm{~Hz}$ and $0.2 \mathrm{rad} / \mathrm{m}$ and (b) '+' at $744 \mathrm{~Hz}$ and $0.2 \mathrm{rad} / \mathrm{m}$ in Fig. 10(a). 
are illustrated in Fig. 11. Fig. 11 shows that these two waves are localized in the two bays in the middle of the bottom plate. Due to the two sets of double vertical stiffeners about $y=0.28 \mathrm{~m}$ and $0.62 \mathrm{~m}$, the vibration of the panel is trapped within the two bays in the mid section.

On the other hand, for the excitation on the stiffener, it can be seen from Fig. 10 that relatively weak vibration and radiation occur compared with the strip excitation. In addition, Fig. 10 shows that the responses are mostly limited to wavenumbers below the vertical bending wavenumber of the equivalent single plate. This implies that the interior stiffeners govern the global rigidity of the extruded panel so that the panel deforms mainly globally by the stiffener excitation. To examine it, the deformation shapes of the two waves, marked with ' $x$ ' and ' + ' in Fig. 10(a), generating the relatively large vibration are illustrated in Fig. 12. Since the excitation is now applied on a vertical stiffener, the two waves have global deformations.

It is observed from Figs 9 and 10 that the excitation on the stiffener causes less vibration and sound radiation than the excitation on the strip. To show this effect more clearly, the average mean-squared velocities and the radiated sound powers integrated over wavenumber at each frequency are compared in Fig. 13 for the two types of excitation. It can be seen from Fig. 13(a) that the excitation on the strip causes much larger vibration than that on the stiffener above about $200 \mathrm{~Hz}$ and the level difference increases above $400 \mathrm{~Hz}$ to almost $30 \mathrm{~dB}$. The strip excitation also generates about $15 \mathrm{~dB}$ greater sound power on

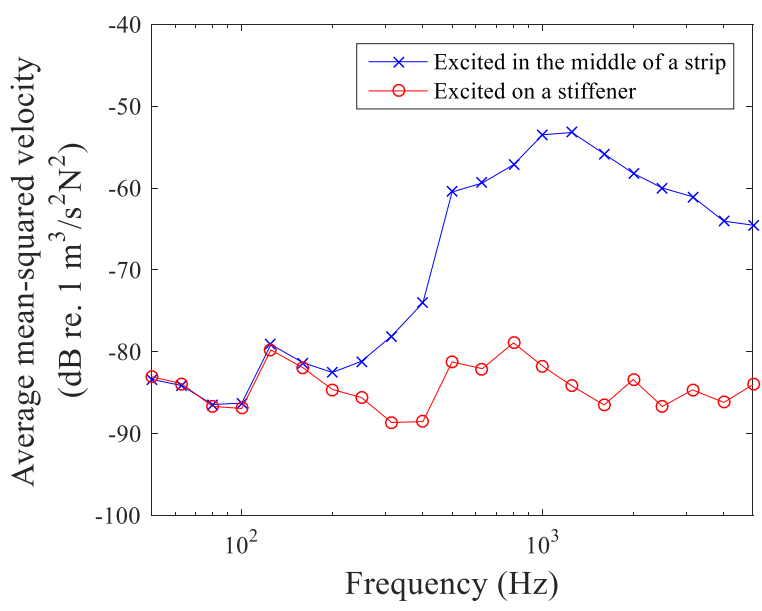

(a)

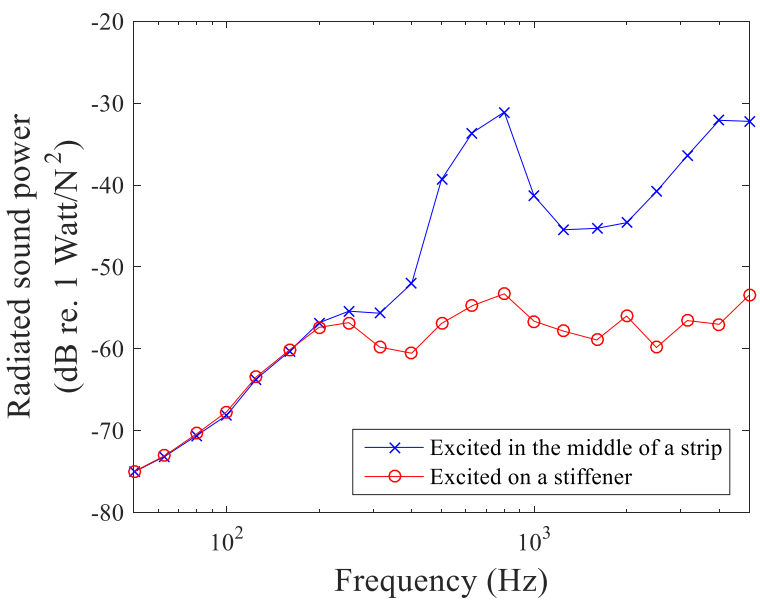

(b)

Fig. 13. Comparison of (a) the average mean-squared velocity and (b) radiated sound power of the panel, predicted from the two different excitations in the frequency domain (integrated over the wavenumber). 


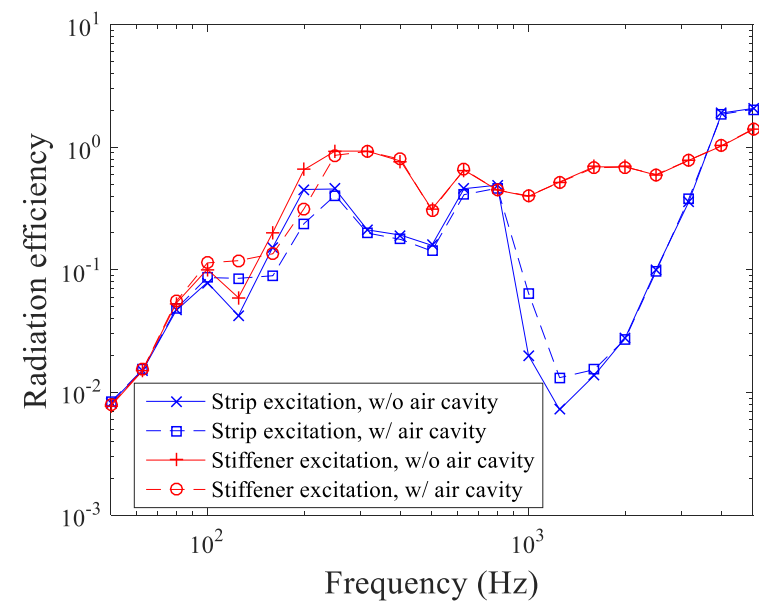

Fig. 14. The predicted radiation efficiencies of extruded panel with and without air-filled cavity.

average than the stiffener excitation above $400 \mathrm{~Hz}$, increasing rapidly from $400 \mathrm{~Hz}$ with a maximum difference of $25 \mathrm{~dB}$ at $630 \mathrm{~Hz}$, as shown in Fig. 13(b). The differences in the averaged velocity are bigger than those in the sound power due to the contribution of the waves located above the acoustic wavenumber.

Radiation efficiencies predicted are presented in Fig. 14 in one-third octave bands for the two different types of excitation. This shows that the strip and stiffener excitations yield a significant variation of radiation efficiency between $1 \mathrm{kHz}$ and $3.15 \mathrm{kHz}$. In this frequency range, the radiation efficiency for the stiffener excitation has levels about 15 20 dB larger than those for the strip excitation. This increase can be explained by the fact that the strong vibration existing above the acoustic wavenumber, which is created by the strip excitation, does not radiate sound into the far field, as indicated in Figs 9 and 10.

So far, the internal spaces filled with air between the top and bottom plates were excluded in the calculation. The effects of the internal air on the radiation efficiency are compared in Fig. 14. In this prediction the loss factor of the air cavity is neglected because the results are only changed by $1 \mathrm{~dB}$ for a loss factor of $10^{-3}$, as reported in [3, 26]. It is found from Fig. 15 that interactions between the panel structure and internal air occur mainly below about $400 \mathrm{~Hz}$ for both excitation positions and around $1 \mathrm{kHz}$ for the strip excitation. Below about $400 \mathrm{~Hz}$, a reduction by up to $3 \mathrm{~dB}$ in the radiation efficiency takes place because global wave modes of the panel interact strongly with the internal acoustic wave, see Fig. 8(a). This coupling effect at low frequencies appears regardless of the excitation positions. On the other hand, around $1 \mathrm{kHz}$, the radiation efficiency increases by about $5 \mathrm{~dB}$ for the strip excitation 


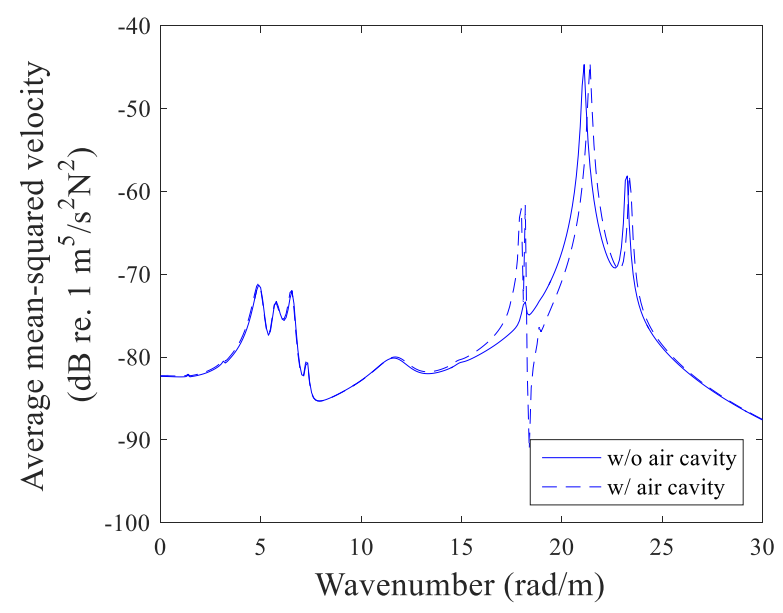

(a)

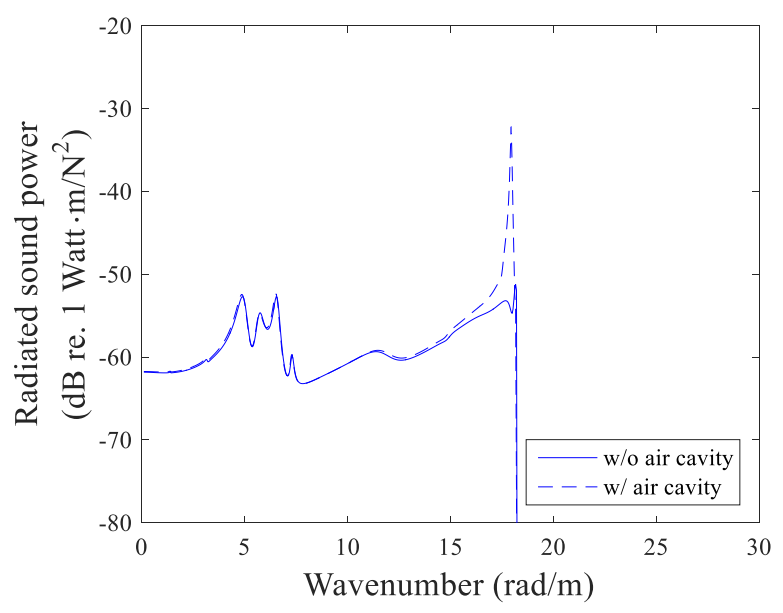

(b)

Fig. 15. (a) The average mean-squared velocity and (b) radiated sound power of the panel with and without air-filled cavity against wavenumber at $1 \mathrm{kHz}$ for the strip excitation.

due to the internal air. To help explain this, Fig. 15 shows the wavenumber domain responses at $1 \mathrm{kHz}$ with and without the air-filled cavity for the strip excitation. Fig. 15(a) shows that there is a considerable change of the velocity due to the internal air cavities around $18 \mathrm{rad} / \mathrm{m}$, which corresponds to the acoustic wavenumber at $1 \mathrm{kHz}$. However, when this is integrated over the whole wavenumber range, the results with and without air cavity become nearly identical. In terms of the radiated power, however, the waves above the acoustic wavenumber do not radiate sound into the far field as seen in Fig. 15(b). Hence, the integral of the radiated power over the wavenumber domain results in a considerable increase of the radiated power. For stiffener excitation, on the contrary, the air cavities do not make a significant variation to the predicted result at high frequencies because the vibration responses are mostly limited to wavenumbers below the vertical bending wavenumber of the equivalent plate. Base on this examination, the averaged mean-squared velocity and radiation efficiencies predicted by including the air cavities will be compared with experimental results in Sec. 5.2.2.

\subsection{STL of the extruded panel}

\subsubsection{Calculation of the STL}

For the calculation of sound transmission using the WFE/BE method, incident waves which excite the panel should be defined, i.e., $\tilde{\mathbf{P}}_{i}$ in Eq. (12). It is assumed that the sound is 


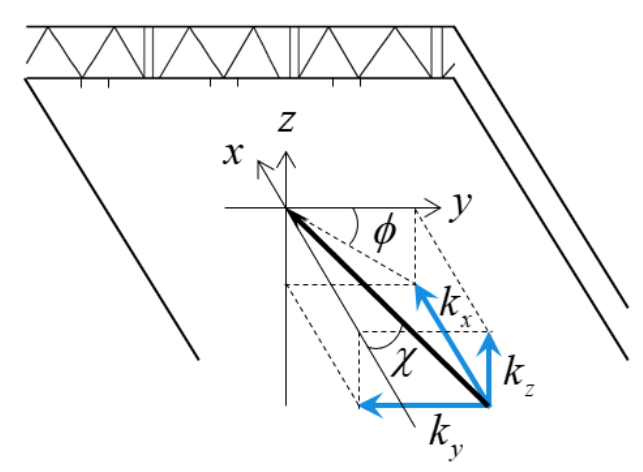

(a)

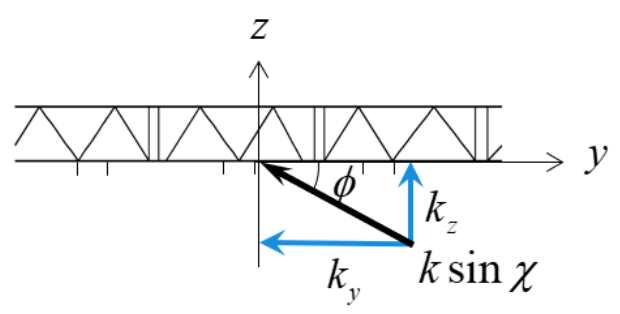

(b)

Fig. 16. Coordinates used to define a wave incident on the bottom plate (a) in 3D space and (b) in the $y-z$ plane.

incident on the bottom plate with an angle, $\phi$, in the $y-z$ plane and an angle, $\chi$, relative to the $x$-axis, as depicted in Fig. 16. The incident sound pressure at a frequency $\omega$ can be written as

$$
p_{i}(x, y, z)=P_{i} \mathrm{e}^{-\mathrm{i} k_{x} x} \mathrm{e}^{-\mathrm{i} k_{y} y} \mathrm{e}^{-\mathrm{i} k_{z} z},
$$

where $P_{i}$ is the amplitude of the incident sound pressure and $k_{x}, k_{y}$ and $k_{z}$ are the wavenumbers along the $x, y$ and $z$ directions, respectively. These can be expressed in terms of the angles $\phi$ and $\chi$ as

$$
k_{x}=k \cos \chi, \quad k_{y}=k \sin \chi \cos \phi, \quad k_{z}=k \sin \chi \sin \phi
$$

where $k_{x}$ is equal to the structural wavenumber, $\kappa$, and the acoustic wavenumber of the incident wave is written as $k=\left(k_{x}^{2}+k_{y}^{2}+k_{z}^{2}\right)^{1 / 2}$. Since the component of fluid particle velocity of the incident wave normal to the bottom plate is given by

$$
v_{i}(x, y)=\frac{p_{i}(x, y)}{\rho_{0} c_{0}} \sin \phi \sin \chi,
$$

the incident power per unit length in the $x$-direction, $\hat{\Pi}_{i}$, can be obtained by

$$
\hat{\Pi}_{i}(\phi, \chi)=\frac{1}{2} \int_{\Gamma} \frac{\left|P_{i}\right|^{2} \sin \phi \sin \chi}{\rho_{0} c_{0}} \mathrm{~d} y .
$$


The sound pressure, $\tilde{p}_{t}(\phi, \chi, y)$, and fluid particle velocity, $\tilde{v}_{t}(\phi, \chi, y)$, on the surface of the top plate can be used to determine the transmitted power per unit length, $\hat{\Pi}_{t}(\phi, \chi)$, given by

$$
\hat{\Pi}_{t}(\phi, \chi)=\frac{1}{2} \operatorname{Re}\left\{\int_{\Gamma} \tilde{p}_{t}(\phi, \chi, y) \tilde{v}_{t}^{*}(\phi, \chi, y) \mathrm{d} y\right\} .
$$

The sound power transmission coefficient, $\tau$, is defined by the ratio between the incident and transmitted powers as

$$
\tau(\phi, \chi)=\frac{\hat{\Pi}_{t}(\phi, \chi)}{\hat{\Pi}_{i}(\phi, \chi)} .
$$

Since Eq. (23) applies to the specific angles $\phi$ and $\chi$, the diffuse field transmission coefficient, $\tau_{d}$, is evaluated using [27]

$$
\tau_{d}=\frac{\int_{\chi_{0}}^{\pi / 2} \int_{\phi_{0}}^{\pi / 2} \tau(\phi, \chi) \sin ^{2} \chi \sin \phi \mathrm{d} \phi \mathrm{d} \chi}{\int_{\chi_{0}}^{\pi / 2} \int_{\phi_{0}}^{\pi / 2} \sin ^{2} \chi \sin \phi \mathrm{d} \phi \mathrm{d} \chi} .
$$

According to ref. $[15,28]$, the range of elevation angles which gives a good agreement between the predicted and measured results is from $12^{\circ}$ to $90^{\circ}$ in the coordinates given in Fig. 16. Hence, the lower limits of the integration range for $\phi$ and $\chi$ are set to $\phi_{0}=\chi_{0}=12^{\circ}$ in this prediction. Finally, the STL through the panel for an incident diffuse sound field is determined using

$$
R=10 \log _{10}\left(\frac{1}{\tau_{d}}\right)
$$

\subsubsection{Application of the spatial window functions for finite length panels}

The WFE/BE approach adopted in the present paper is based on the assumption that the model is infinitely long in the $x$ direction. For practical applications to structures with a finite length, however, this assumption is not suitable. In general, the STL of a panel is evaluated in experiments by installing it in an aperture in a rigid wall located between two chambers. This corresponds to a baffled condition along the $x$ direction of the panel. To represent this in the WFE/BE method, a rectangular window function is introduced here corresponding to the length of the actual panel. 
A Hanning window, which gives zero displacements at the boundaries, may be more appropriate than a rectangular window for the comparison with the measured results. However, it was confirmed that the STL with a Hanning window is only approximately $2 \mathrm{~dB}$ higher than that with a rectangular window below $400 \mathrm{~Hz}$ for the present model. Hence, only the STL with the rectangular window will be presented in this paper. Windowing was not applied to the prediction of the radiation efficiency in the previous section because in the corresponding measurement the baffled boundary condition along the $x$ direction is not satisfied (see Sec. 5.2.1).

A rectangular window function in the spatial domain is applied to the infinitely long waveguide structure, given by $w(x)=1$ for $|x|<L_{w} / 2$ and $w(x)=0$ for elsewhere. $L_{w}$ denotes the length of the window function which corresponds to the length of the finite panel. This rectangular window can be represented in the wavenumber domain by the Fourier transform as

$$
\tilde{w}(\kappa)=L_{w} \frac{\sin \left(\kappa L_{w} / 2\right)}{\kappa L_{w} / 2} .
$$

The wavenumber domain window function in Eq. (26) is used for calculating the sound transmission in this study.

The entire process of implementing spatial windows can be decomposed into three steps [16]. As a first step, the window function is applied to the incident pressure which impinges with the angles $\phi$ and $\chi$ in the spatial domain by

$$
p_{i, w}(x, y, \phi, \chi)=p_{i}(x, y, \phi, \chi) w(x),
$$

where the subscript $w$ denotes the windowed quantity. Eq. (27) can be expressed as a convolution in the wavenumber domain

$$
\tilde{p}_{i, w}(\kappa, y, \phi, \chi)=\tilde{p}_{i}(\kappa, y, \phi, \chi) * \tilde{w}(\kappa),
$$

where the operator $*$ denotes the convolution. The windowed fluid particle velocity of the incident plane wave is found from $\tilde{p}_{i, w}$ by

$$
\tilde{v}_{i, w}(\kappa, y, \phi, \chi)=\frac{\tilde{p}_{i, w}(\kappa, y, \phi, \chi)}{\rho_{0} c_{0}} \sin \phi \sin \chi .
$$

Then, the windowed incident power, $\Pi_{i, w}(\phi, \chi)$, can be obtained by 


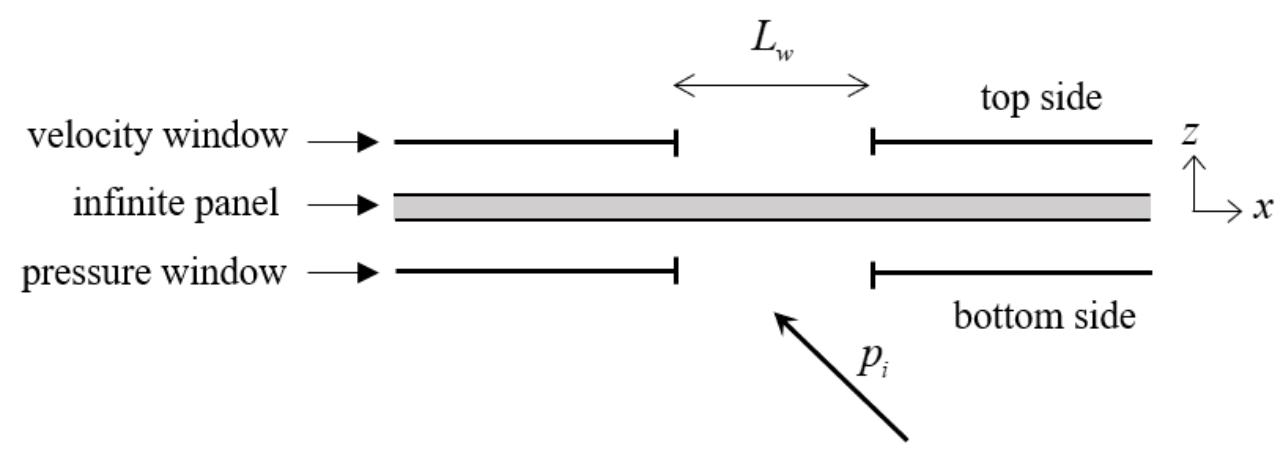

Fig. 17. The spatial windowing effects on the incident and transmitted sides of the panel.

$$
\Pi_{i, w}(\phi, \chi)=\frac{1}{4 \pi} \operatorname{Re}\left\{\int_{-k}^{k} \int_{\Gamma} \tilde{p}_{i, w}(\kappa, y, \phi, \chi) \tilde{v}_{i, w}^{*}(\kappa, y, \phi, \chi) \mathrm{d} y \mathrm{~d} \kappa\right\} .
$$

As the second step, the transmitted power by the windowed incident wave, $\Pi_{t, w}$, can be obtained from the WFE/BE solutions $\left(\tilde{v}_{t, w}\right.$ and $\left.\tilde{p}_{t, w}\right)$. The ratio between $\Pi_{i, w}(\phi, \chi)$ and $\Pi_{t, w}(\phi, \chi)$ for the infinite panel has already been evaluated as $\tau(\phi, \chi)$ by Eq. (23). In the third step, the second window function (velocity window in Fig. 17) is applied to the panel's velocity at the transmitting side. After finding the windowed normal velocity, $\tilde{v}_{t, w w}$, of the top panel, the transmitted sound pressure, $\tilde{p}_{t, w w}$, can be calculated by

$$
\tilde{p}_{t, w w}(\kappa, y)=-\mathrm{i} \omega \rho_{0} \mathbf{H}^{-1} \mathbf{G} \tilde{v}_{t, w w}(\kappa, y),
$$

and finally the power transmitted through the finite length panel, $\Pi_{t, w w}(\phi, \chi)$, can be predicted by integration as in Eq. (30).

Hence, the transmission coefficient, $\tau_{w}$, for the windowed panel can be expressed as a combination of the three steps by

$$
\tau_{w}(\phi, \chi)=\frac{\Pi_{i, w}(\phi, \chi)}{\Pi_{i}(\phi, \chi)} \tau(\phi, \chi) \frac{\Pi_{t, w w}(\phi, \chi)}{\Pi_{t, w}(\phi, \chi)},
$$

where $\Pi_{i}=L_{w} \hat{\Pi}_{i}$. Eq. (32) shows that the windowed transmission coefficient can be calculated from the product of the transmission coefficient of the infinite panel, $\tau(\phi, \chi)$, and the ratios between the powers before and after applying the window functions. The two terms $\Pi_{i}(\phi, \chi)$ and $\Pi_{i, w}(\phi, \chi)$ for the incident side in Eq. (32) can be found theoretically, whereas the remaining three parameters $\left(\tau(\phi, \chi), \Pi_{t, w}(\phi, \chi)\right.$ and $\left.\Pi_{t, w w}(\phi, \chi)\right)$ are 
evaluated from the WFE/BE analysis. For diffuse incidence, the STL of the windowed panel can be obtained by following the calculation given in Eqs (24) and (25), replacing $\tau$ with $\tau_{w}$ in Eq. (24).

\subsubsection{Predicted results for the STL}

To check the convergence of the result for the number of incident angles, the STLs for two different numbers of incident angles are compared. The angles $\phi$ and $\chi$ are both set to vary from $12^{\circ}$ to $90^{\circ}$ with 15 or 25 equally spaced steps so that 225 or 625 incident angles in total are considered. It was found from this comparison that the STL remains nearly the same for these two different intervals of the angles. Based on this result, 225 incident angles with 15 equally spaced intervals are used in the remainder of this study. The calculation time is about 25 seconds per frequency using the same computer used in the calculation of radiation efficiency. If the internal air cavity is considered in the calculation, the time is again increased by about a factor of three.

The STLs with and without air cavities between the two outer plates are also compared. This examination showed that the air cavities do not make a considerable change to the predicted STL in the whole frequency range. This can be explained from the influence of the internal fluid on the dispersion relations, see Figs 4(b) and 6(b). The structural wavenumbers in the extruded panel remain lower than the acoustic wavenumbers below 350 Hz. This implies that the air cavities do not interact strongly with the structural waves at low frequencies. Moreover, at high frequencies the coupling with the internal fluid will be relatively weak because of the strong mechanical connection between the top and bottom panels. Consequently, this ensures that the effects of the air cavity on the sound transmission is negligible for this complex-shaped panel. Based on this result, the air cavities are disregarded in the further calculations to make the model small and to save computing times. In the case of a double layered panel having less structural connections between the two plates, the effects of the air cavities would become significant [3], leading to the so-called mass-air-mass resonance [28].

The STL predicted for the simply supported boundary conditions is presented in Fig. 18(a). In order to see the contribution of each wave to sound transmission, the transmission coefficient $\tau(\chi)$ is evaluated for each frequency and illustrated in Fig. 18(b) as an image

plot over frequency-wavenumber domain by using $k_{x}=k \cos \chi \cdot \tau(\chi)$ in Fig. 18(b) is 


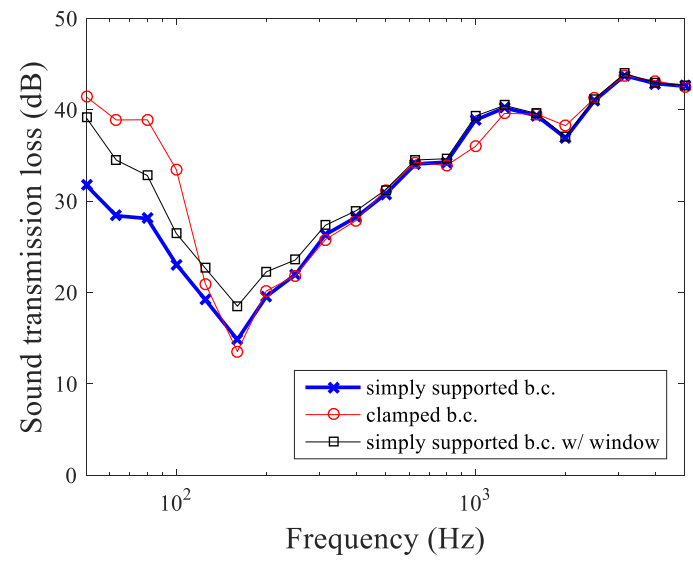

(a)

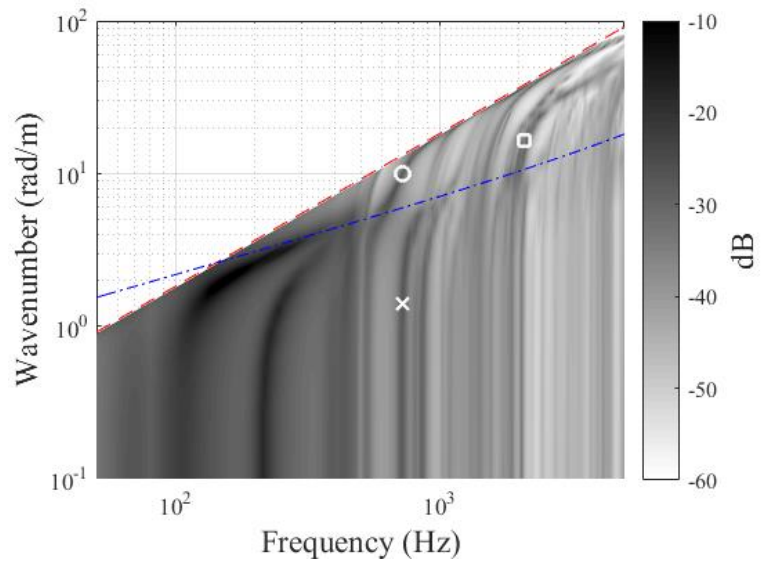

(b)

Fig. 18. (a) Predicted STLs through the extruded panel and (b) image plot of the transmission coefficient $\tau(\chi)$ for simply supported boundary conditions against frequency and wavenumber.

calculated by performing only a single integration over $\phi$ in Eq. (24). The vertical bending wave curve of the equivalent plate is also plotted in Fig. 18(b).

Fig. 18(a) shows that the lowest STL (i.e., the largest transmission in Fig. 18(b)) occurs at $150 \mathrm{~Hz}$ but the first cut-on frequency of the simply supported panel is about $87 \mathrm{~Hz}$ as shown in Fig. 4(b). In general, simple panels having a constant damping over frequency have the minimum STL at the first cut-on frequency of the global motion but the extruded panel does not due to the high damping at this frequency (see Fig. 3). Fig. 18(b) reveals that the sound generated from the global wave mode is transmitted well at low frequency below

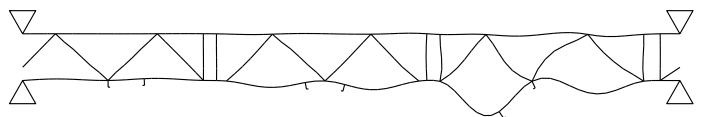

(a)

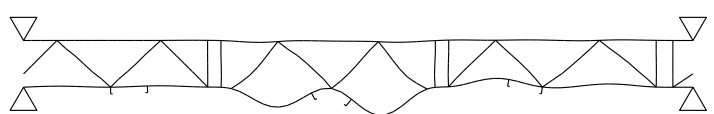

(c)

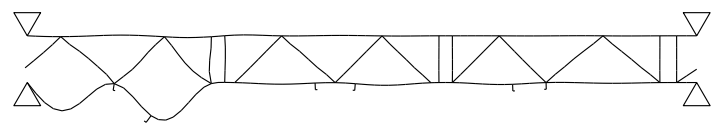

(b)

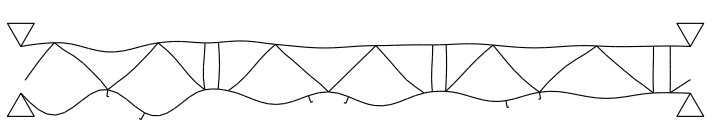

(d)

Fig. 19. (a), (b) and (c) The deformation shapes of the three waves marked with ' $\bigcirc$ ' at 724 $\mathrm{Hz}$ and $10 \mathrm{rad} / \mathrm{m}$ and (d) a wave marked with ' $x$ ' at $724 \mathrm{~Hz}$ and $1.4 \mathrm{rad} / \mathrm{m}$ in Fig. $18(b)$. 

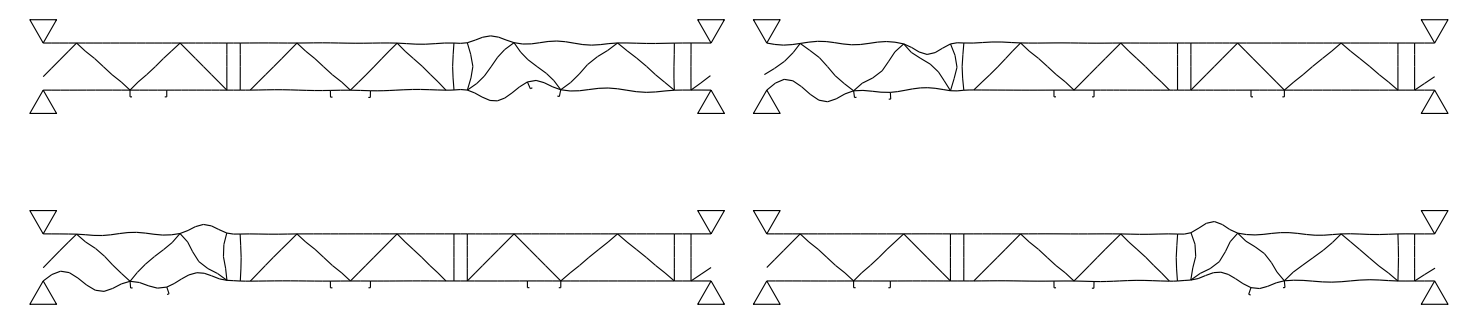

Fig. 20. The deformation shapes of the waves around $2 \mathrm{kHz}$ and $16.6 \mathrm{rad} / \mathrm{m}$, marked with ' $\square$ ', in Fig. 18(b).

$400 \mathrm{~Hz}$. Also, there are several waves which make distinguishable transmission around 800 $\mathrm{Hz}$ and $2 \mathrm{kHz}$, corresponding to the dips in Fig. 18(a).

To check the wave modes contributing to the dip of STL around $800 \mathrm{~Hz}$ in Fig. 18(a), the waves marked with ' $\bigcirc$ ' and ' $x$ ' at $724 \mathrm{~Hz}$ in Fig. 18(b) are chosen. At the wavenumber of $10 \mathrm{rad} / \mathrm{m}$, marked with ' $O$ ', three wave curves overlap as presented in Fig. 4(b). The deformation shapes of these three waves are illustrated in Figs 19(a), 19(b) and 19(c). All of them are the waves localised in each one-third section of the panel and have the first order modes of the two individual bays in there. Since these waves are located above the vertical bending wavenumber of the equivalent plate, it is also justified that they are said to localized waves. Meanwhile, there exists two waves at $1.4 \mathrm{rad} / \mathrm{m}$ (marked with ' $x$ '), which is below the vertical bending wave curve of the equivalent plate. The deformation shape of one of the two waves are shown in Fig. 19(d). This wave has the global deformation of the top and bottom plates but each bay has the first order mode.

Another dip of STL at $2 \mathrm{kHz}$ in Fig. 18(a) is caused mainly by the waves around $16.6 \mathrm{rad} / \mathrm{m}$ marked with ' $\square$ ' in Fig. 18(b). Five waves exist there, and the deformation shapes of four of them are illustrated in Fig. 20. They are the waves having the localized second order mode of the bays in the bottom plate and the first order mode of the narrow bays in the top plate. It is found from Figs 18, 19 and 20 that the STL drops due to the local resonances of the bays in the extruded panel.

In order to see the effect of the boundary conditions on the STL, the fixed boundary conditions are applied at both ends of $y=0$ and $1 \mathrm{~m}$. The dispersion diagram of the extruded panel with the fixed boundary conditions are illustrated in Fig. 21(a). It is found from Fig. 21(a) that the first global wave mode cuts on at $136 \mathrm{~Hz}$, which is higher than for the 


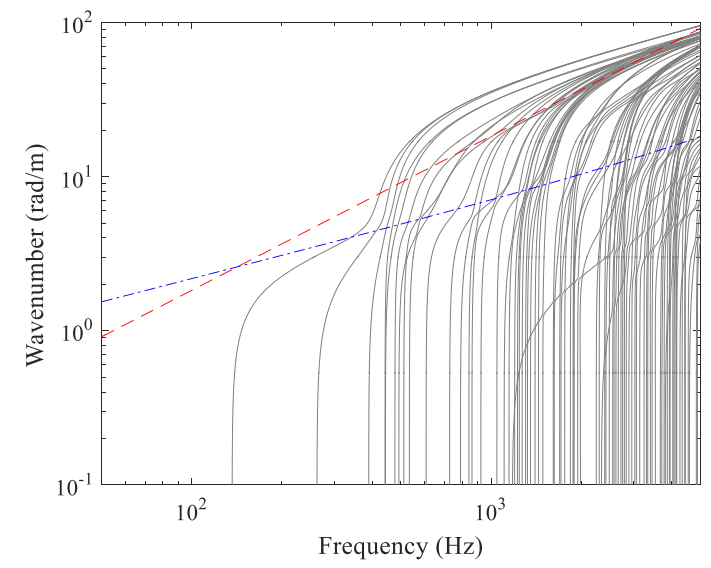

(a)

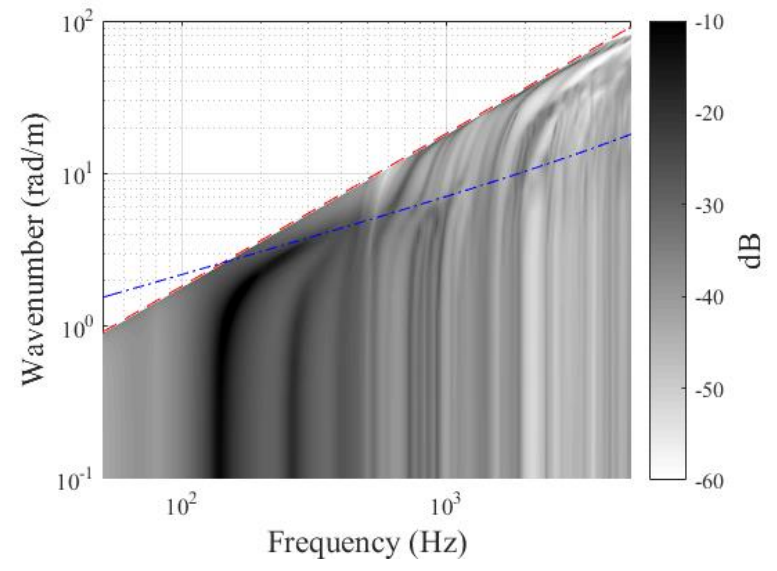

(b)

Fig. 21. (a) The dispersion diagram for the fixed boundary condition and (b) image plot of the transmission coefficient $\tau(\chi)$ for fixed boundary conditions against frequency and wavenumber.

simply supported boundary condition in Fig. 4(b) but the dispersion curves for these two different boundary conditions become similar as frequency increases. The STL predicted for the fixed boundary condition is illustrated in Fig. 18(a) together with that for simply supported boundary conditions. Fig. 18(a) shows that there is an increase of STL up to about $10 \mathrm{~dB}$ at frequencies below $125 \mathrm{~Hz}$, where the first global wave mode cuts on with the fixed boundary condition. However, the variation in the predicted results due to the boundary conditions is negligible above $125 \mathrm{~Hz}$, except around $1 \mathrm{kHz}$ where about $3 \mathrm{~dB}$ drops due to the fixed boundary condition. Also, they still have two dips at $800 \mathrm{~Hz}$ and $2 \mathrm{kHz}$.

To see the wavenumber domain feature, the transmission coefficient $\tau(\chi)$ is presented in Fig. 21(b) as an image plot. In comparison between Figs 18(b) and 21(b), it is seen that the panel with the fixed boundary condition transmits more power around $900 \mathrm{~Hz}$ than that with the simply supported boundary conditions because there are a couple of more waves around that frequency. Hence, the STL for fixed boundary conditions becomes about 3 $\mathrm{dB}$ lower than that for simply supported boundary conditions, as shown in Fig. 18(a). Since, in the experiment, the actual fixture condition would be in between the simply supported and fixed boundary conditions, the STLs for the both boundary conditions will be compared with the experimental results in Sec. 5.3.2.

The effect of the spatial window on the STL is also examined and illustrated in Fig. 18(a). This investigation shows that the rectangular window increases the STL by up to about 
$7 \mathrm{~dB}$ at frequencies below about $400 \mathrm{~Hz}$, where the global wave modes contribute dominantly. However, the applied window function makes negligible effects to the predicted STL above $400 \mathrm{~Hz}$. This implies that an extruded panel with a finite length can be regarded as infinitely long at frequencies above the cut-on of the strip wave modes. The predicted STL using the spatial window will be compared with the experimental results in Sec. 5.3.2.

\section{Comparison of numerical results with experiments}

In this section, the results obtained from the WFE/BE approach are compared with those obtained experimentally on a finite panel. The extruded panel used in the experiment is shown in Fig. 2(a) and has a length of $1.5 \mathrm{~m}$ along the extrusion. Regarding the actual length of the train coaches and the assumption of waveguide, the length $1.5 \mathrm{~m}$ would not be long enough but chosen for lab experiments. The experiments for the radiation efficiency and sound transmission were done separately with different set-ups.

\subsection{Comparison of point mobility}

In this section, the predicted point mobility of the extruded panel is compared with measured ones obtained by Zhang et al. [9], who hung the panel freely with elastic ropes and measured the point mobilities using an impact hammer and an accelerometer. The measured positions are illustrated in Fig. 22. S1 to S4 are located midway between two adjacent interior and exterior stiffeners on the bottom side, except S2 which is off-centre on a strip. S5 to S10 are midway between two adjacent interior stiffeners on the top side. All these positions are located at about $1 / 5$ of panel length from one end.

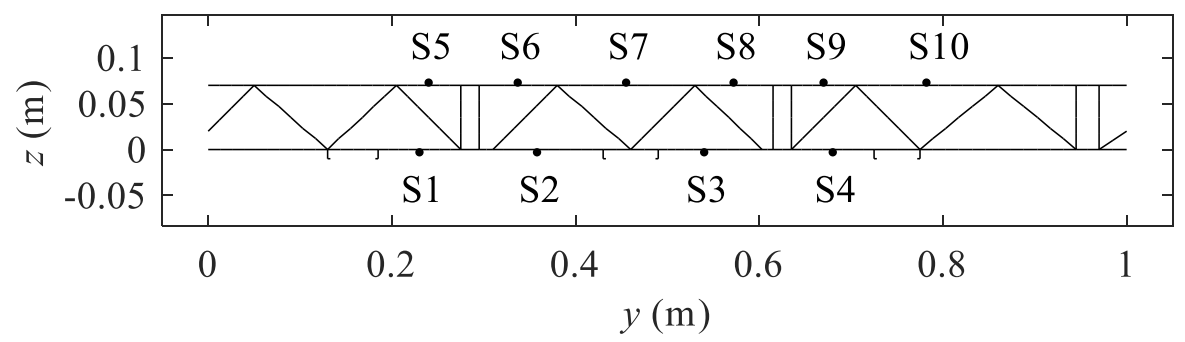

Fig. 22. The cross section of the panel and measured points for the point mobilities [9]. 


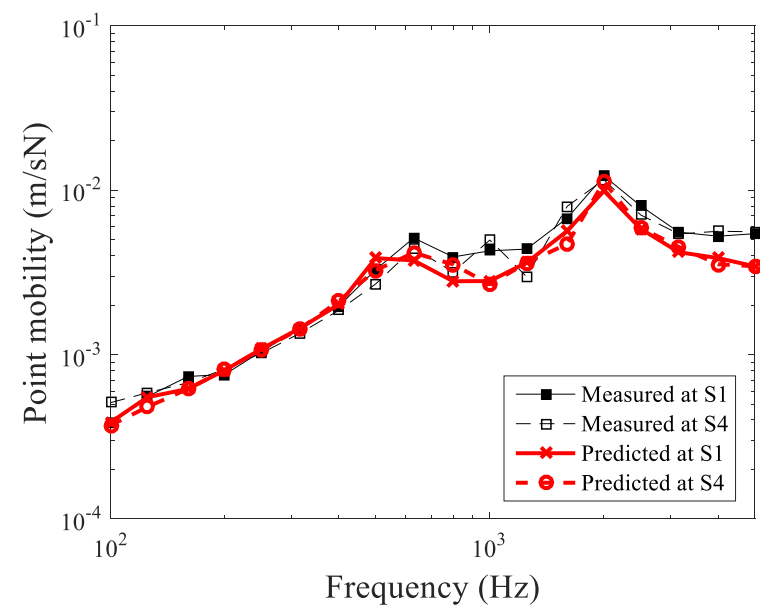

(a)

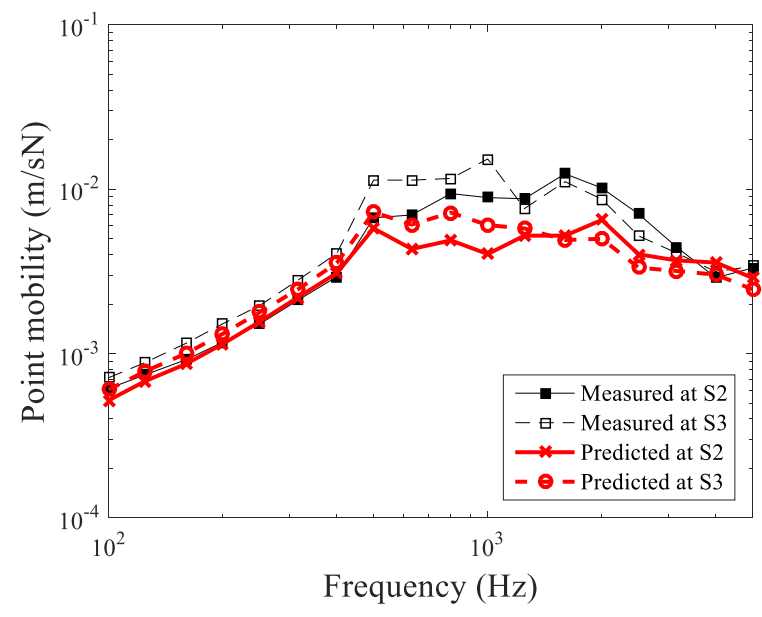

(b)

Fig. 23. Comparison of point mobilities obtained from numerical simulation and experiments at (a) S1 and S4, and (b) S2 and S3 on the bottom plate.

The point mobilities at S1 and S4 are illustrated together in Fig. 23(a) because they are in similar geometric conditions (width of bay and location of exterior stiffener). The predicted results at these two points agree well with the measured ones in the whole frequency range. The point mobilities in Fig. 23(a) have two peaks around $600 \mathrm{~Hz}$ and $2 \mathrm{kHz}$, coming from the cut-on of the first and second local wave modes of the corresponding bays formed by the two adjacent internal stiffeners, respectively. On the other hand, point mobilities measured and predicted at S2 and S3 are compared in Fig. 23(b). The predicted ones coincide well with the measured ones below $400 \mathrm{~Hz}$ but become about $8 \mathrm{~dB}$ less between $500 \mathrm{~Hz}$ and $2.5 \mathrm{kHz}$ but its reason is not clearly identified yet.

The predicted point mobilities at S5 to S10 on the rubber mat side (top plate) are compared with the measured ones in Fig. 24, grouping them based on analogous geometric condition of the measurement positions. The predicted and measured results at S5 and S9 are presented in Fig. 24(a). Since S5 and S9 are in nearly the same geometric condition (strip width and the location within the strip), the point mobilities at these two points are expected to be similar. However, the measured result at S5 have a large discrepancy below $315 \mathrm{~Hz}$ so that it seems to have a measurement error there. The numerical and measured results at S6 and S8 in Fig. 24(b) and at S7 and S10 in Fig. 24(c) show good agreements. The frequencies at which the peak responses occur in Fig. 24 correspond to the cut-on of the first order bending wave modes of each bay where the measurement points belong to. 


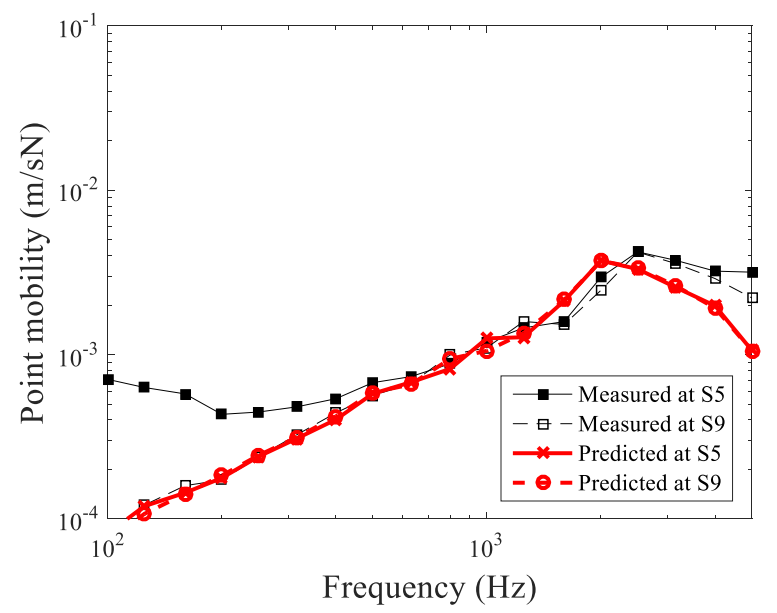

(a)

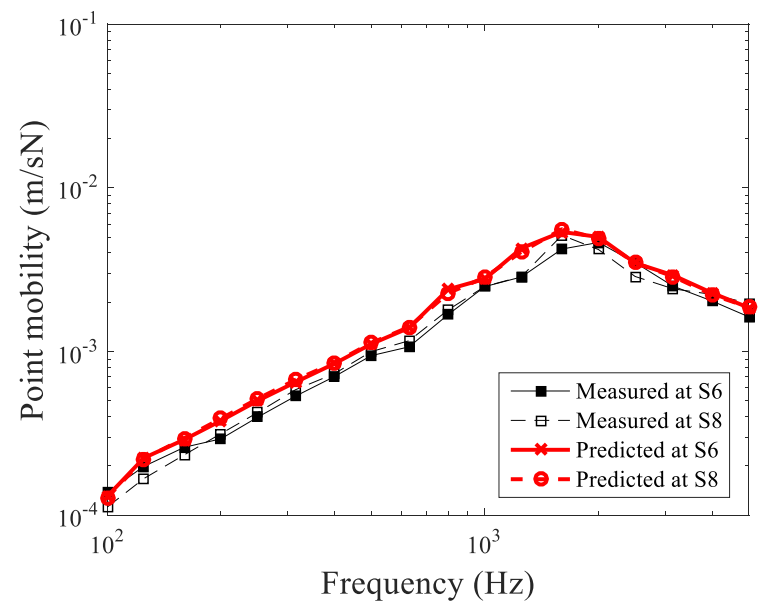

(b)

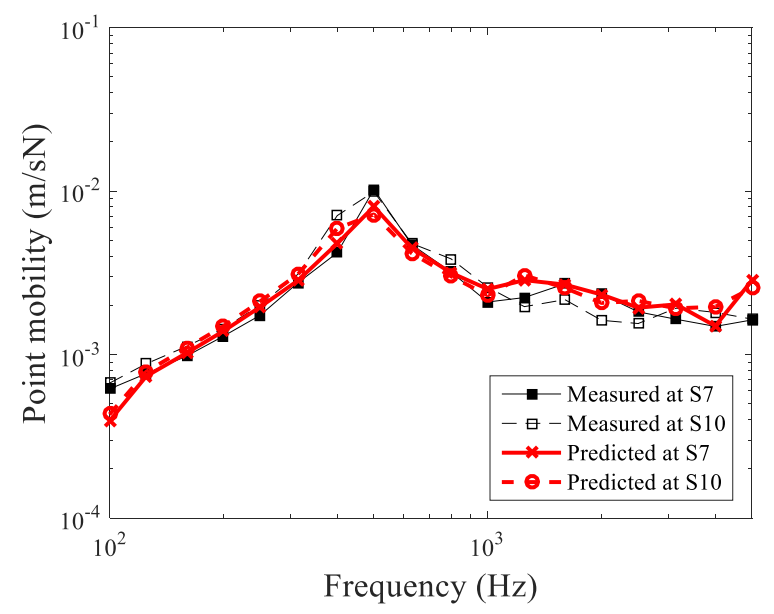

(c)

Fig. 24. Comparison of point mobilities obtained from numerical simulation and experiments at (a) S5 and S9, (b) S6 and S8, and (c) S7 and S10 on the top plate.

\subsection{Comparison of radiation efficiency}

\subsubsection{Experimental set-up of radiation efficiency}

To measure the radiation efficiency, the panel was hung freely with elastic ropes in a reverberation chamber [18] so that that the coupling effects between the panel and chamber would be excluded. A mechanical excitation was applied with a shaker. This shaker was attached to the panel via a slender rod stinger connected to the force transducer. This was necessary to allow the panel to be excited in only one direction so that no rotation was introduced. The measurements were made of the surface-averaged panel vibration using a 


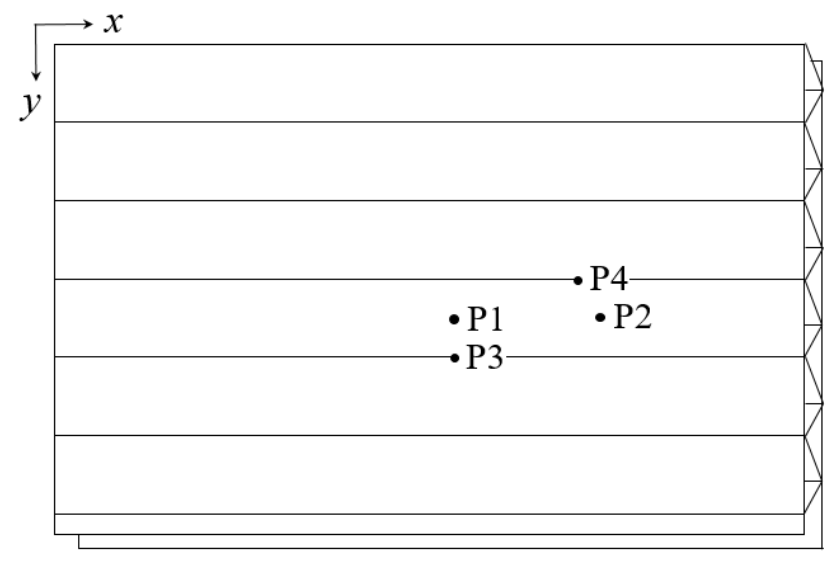

Fig. 25. The locations of four excitation points applied on the bottom plate.

Table 2. The coordinates of excitation points represented in Fig. 25. (The origin is at the top left-hand corner of the plate.)

\begin{tabular}{ccc}
\hline Excitation point & $x(\mathrm{~m})$ & $y(\mathrm{~m})$ \\
\hline P1 & 0.78 & 0.528 \\
P2 & 1.10 & 0.528 \\
P3 & 0.78 & 0.635 \\
P4 & 1.05 & 0.460 \\
\hline
\end{tabular}

scanning laser vibrometer. The spatially-averaged sound pressure in the reverberation chamber was measured, from which the sound power could be determined.

In these measurements, two types of excitation point were chosen on the bottom plate as described in Sec. 4.2.2. One is in the middle of a bay (or a strip), and the other is where an interior stiffener is connected. The specific locations of each excitation point are indicated in Fig. 25, and their coordinates are listed in Table 2.

\subsubsection{Results of radiation efficiency}

In the experiment for the radiation efficiency, the first bay of the bottom plate from the left were excluded in measuring the average mean-squared velocity because the panel was hung there by a wire. To reflect the reduced scanned area in the calculation of the radiation efficiency, the width of $0.87 \mathrm{~m}$, excluding the portion of the first bay of the bottom plate on the left from the panel, is considered at top and bottom plates. The reduced width is also considered in the calculation of the radiated powers because there are few changes of the radiated powers by the reduced width. 
The numerical results of the averaged mean-squared velocity of the panel are compared with experimental ones in Fig. 26. It is stated in ref. [18] that the $x$ directional modes of the extruded panel occur below $400 \mathrm{~Hz}$. The resonance frequencies of the finite length panel are listed in Table 3. $m$ and $n$ in Table 3 denote the orders of the modes in the $x$ and $y$ directions. Since these $x$ directional modes do not appear in the numerical results, there are considerable discrepancies between the predicted and measured results at these resonance frequencies below $400 \mathrm{~Hz}$. For the strip excitation shown in Fig. 26(a), the predicted result shows fair agreement with the measured ones but there is a level drop of about $10 \mathrm{~dB}$ at around $500 \mathrm{~Hz}$. For the stiffener excitation shown in Fig. 26(b), the predicted results are much less than the measured ones in whole frequency range. This increased discrepancy in the stiffener excitation seems to be caused by the fact that the stiffener excitation creates mainly global wave modes which have long wavelength and then easily affected by the finite length.

Fig. 27 shows numerical results of the vibration transmissibility, i.e. the ratio between the average mean-squared velocities on the top and bottom plates. Since the global resonance

Table 3. The resonance frequencies corresponding to the global wave modes [18].

\begin{tabular}{ccccc}
\hline Mode $(m, n)$ & $(1,1)$ & $(2,0)$ & $(2,1)$ & $(1,2)$ \\
\hline Frequency $(\mathrm{Hz})$ & 154 & 190 & 312 & 384 \\
\hline
\end{tabular}

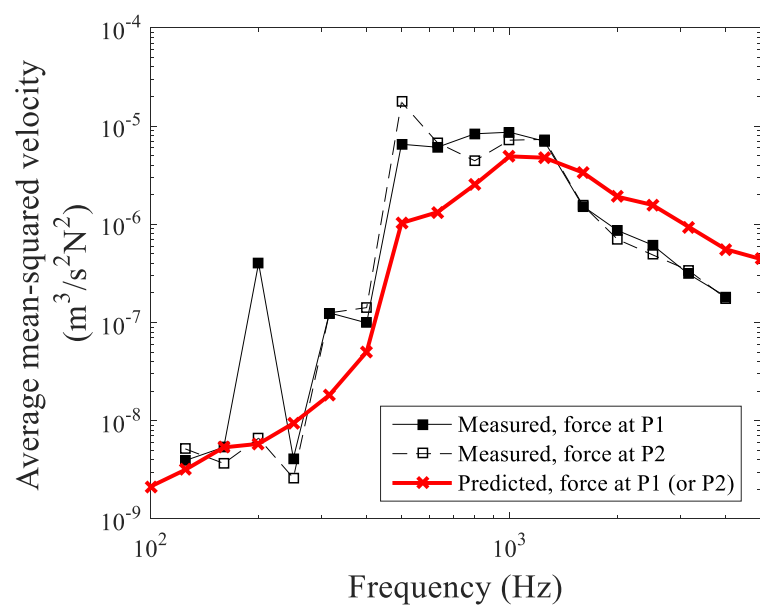

(a)

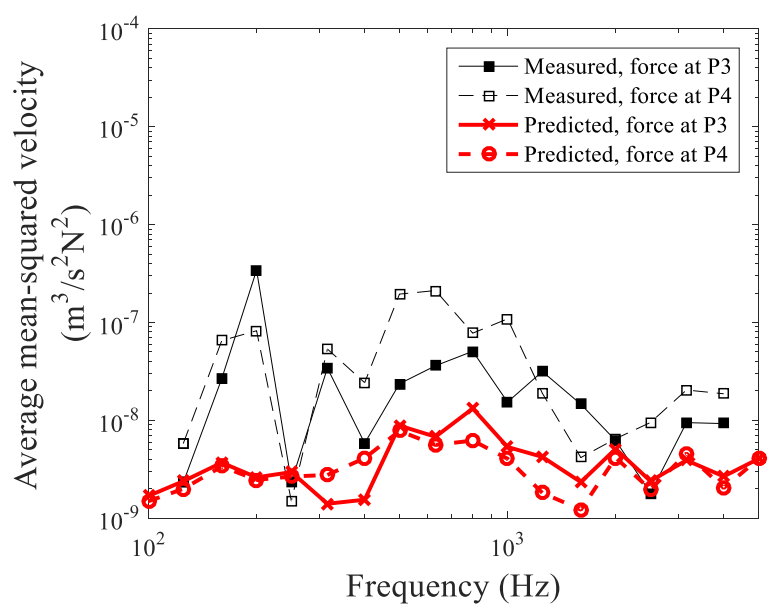

(b)

Fig. 26. Comparison of averaged mean-squared velocity obtained from numerical simulation and experiments for (a) strip excitations at P1 and P2 and (b) stiffener excitations at $\mathrm{P} 3$ and $\mathrm{P} 4$. 


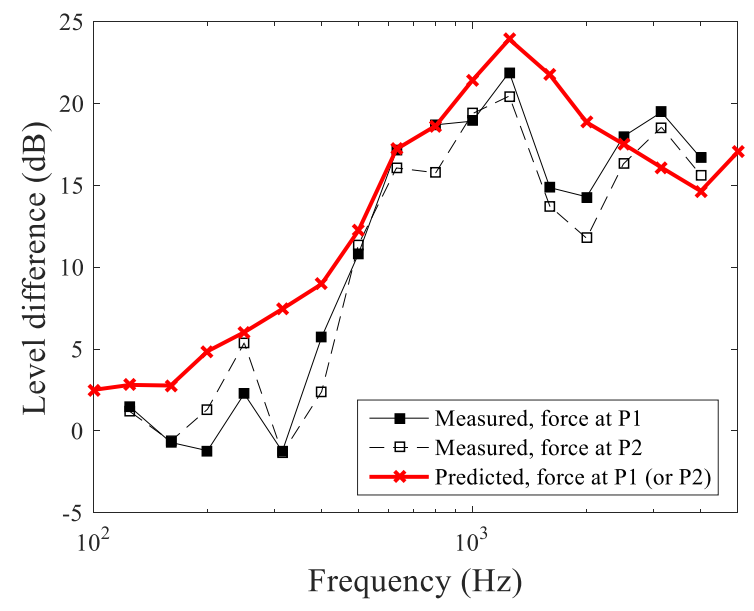

(a)

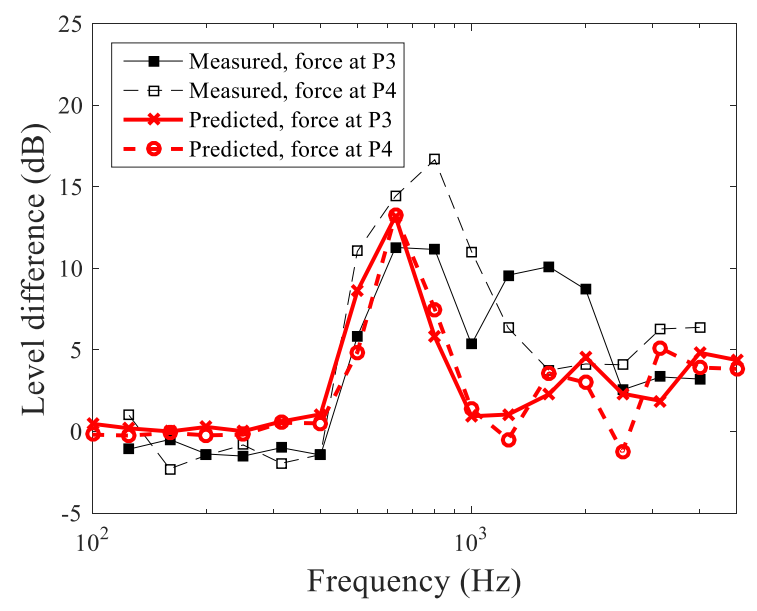

(b)

Fig. 27. Comparison of the vibration level difference between the top and bottom plates obtained from numerical simulation and experiments for (a) strip excitations at P1 and P2 and (b) stiffener excitations at P3 and P4.

modes in Table 3 will have similar level of vibration on the top and bottom plates, the $x$ directional resonances disappear in the vibration transmissibility. For the strip excitation, the vibration transmissibility simulated increases above $400 \mathrm{~Hz}$ where the local strip wave modes occur. This tendency also appears in the experimental results. However, the numerical and measured results have level differences of about $7 \mathrm{~dB}$ around $1.25 \mathrm{kHz}$. On the other hand,

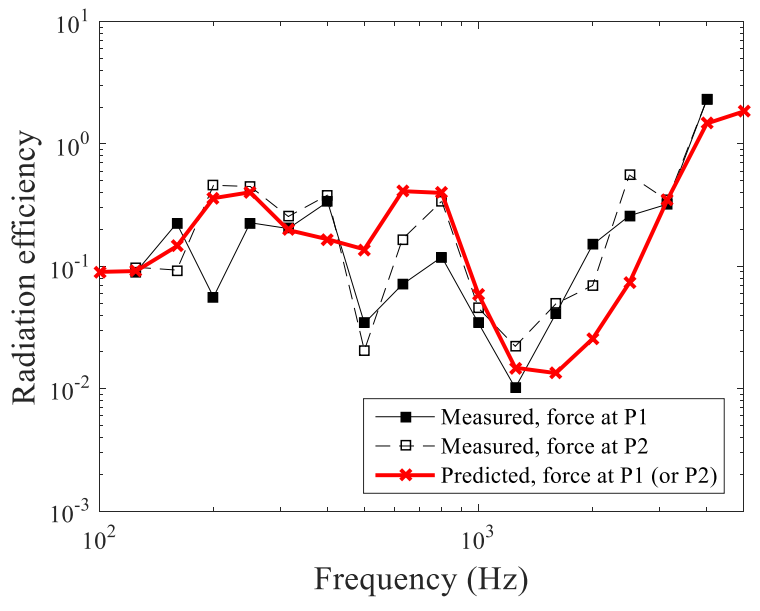

(a)

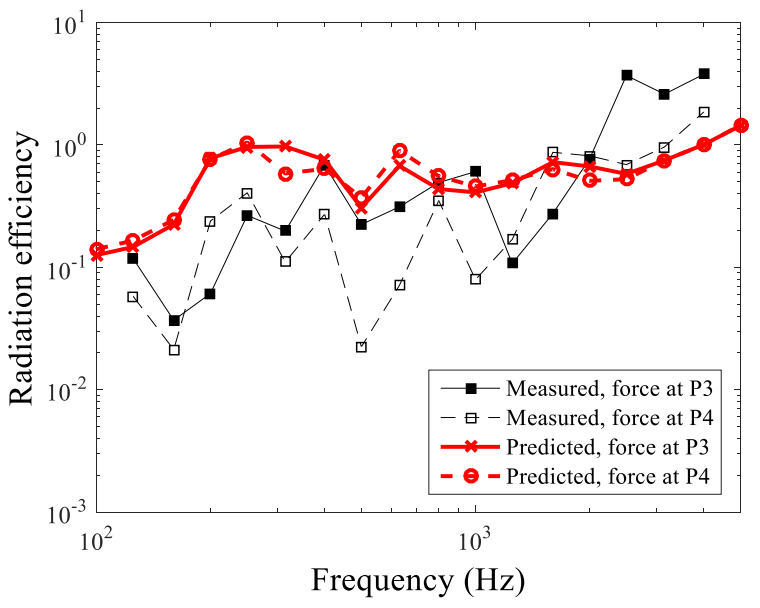

(b)

Fig. 28. Comparison of radiation efficiencies obtained from numerical simulation and experiments for (a) strip excitations at P1 and P2 and (b) stiffener excitations at P3 and P4. 
for the stiffener excitation, the predicted vibration transmissibilities are within about $5 \mathrm{~dB}$ on average except between $400 \mathrm{~Hz}$ and $1 \mathrm{kHz}$ where the bottom plate has bigger deformations than the top plate (see Fig. 12). The measured results in Fig. 27(b) give similar behaviours to the predicted ones but have at most $10 \mathrm{~dB}$ larger transmissibility between $600 \mathrm{~Hz}$ and 2.5 $\mathrm{kHz}$. This implies that the vibration of the bottom plate is underestimated in the simulation. Some of these differences may be caused by the fact that the measurements were conducted on a finite panel whereas the model is for an infinite one.

The measured radiation efficiencies are compared with the predicted ones in Fig. 28. For the strip excitation, the results generally look similar although there are considerable gaps around $500 \mathrm{~Hz}$ and between $1.25 \mathrm{kHz}$ and $2.5 \mathrm{kHz}$. This discrepancy looks coincide with the level of discrepancy in the mean-squared velocity shown in Fig. 26(a), which becomes a denominator in the calculation of the radiation efficiency. Nevertheless, it can be said that the WFE/BE approach is applicable in predicting the radiation efficiency of the finite length panel for the strip excitation. On the other hand, for the stiffener excitation, the predicted results in Fig. 28(b) provide bigger discrepancy than the strip excitation. This may be attributed to the fact that the stiffener excitation generates mainly low wavenumber waves as shown in Fig. 10. Hence, the responses predicted by the WFE/BE approach for the stiffener excitation are likely to be discrepant from those measured from the short finite length panel. Therefore, the WFE/BE method becomes less suitable for the prediction of radiation efficiency from this finite length panel in case of the stiffener excitation.

\subsection{Comparison of STL}

\subsubsection{Experimental set-up of STL}

The experiment for the sound transmission through the extruded panel was conducted according to the International Standard, ISO 140 part 3 [29]. For acoustic excitation, the

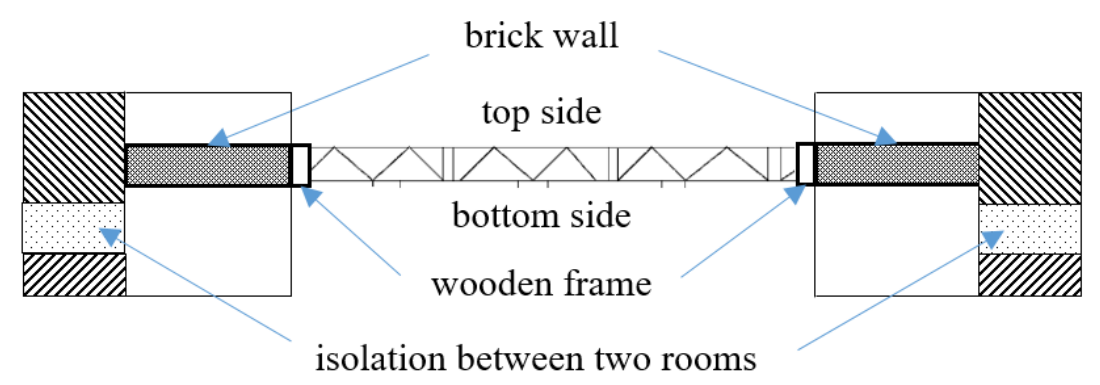

Fig. 29. The cross-section of the extruded panel mounted between two rooms. 
diffuse sound field in the source room was implemented by a loudspeaker driven by random white noise. The diaphragm of loudspeaker faced towards a wall to reduce the contribution of direct sound waves at the microphone. The panel was mounted between two reverberation chambers within a wooden frame and the exposed edges were sealed with silicone rubber as illustrated in Fig. 29. One room was excited using a loudspeaker and the spatially-averaged sound pressures in each room were measured using a pressure microphone attached to a rotating boom. Further details for the experimental set-ups and post-processing of the results are given in ref. [18].

\subsubsection{Result of STL}

For the same extruded panel, Nilsson et al. carried out an experiment to measure the STL by mounting it between an anechoic and reverberation chambers [19]. Comparisons are made with this result as well as the one from [18] measured between two reverberant chambers. In the WFE/BE analysis, two STLs are evaluated for simply supported and fixed boundary conditions by applying the spatial rectangular window function. In the STL prediction, the damping loss factors in Fig. 3 measured from the free panel are again used although these may differ from the actual damping of the panel mounted in the wall, especially at low frequencies. However, no measurements were made of the damping in this mounted configuration.

The predicted and measured STLs are compared in Fig. 30 together with that estimated by the field incidence mass law [28]. The STL measured from ref. [19] is about 2 $\mathrm{dB}$ higher than that in the measurement from ref. [18] above $300 \mathrm{~Hz}$. The STL from the mass law is over $5 \mathrm{~dB}$ higher than the measured ones at low frequencies and the gaps gradually

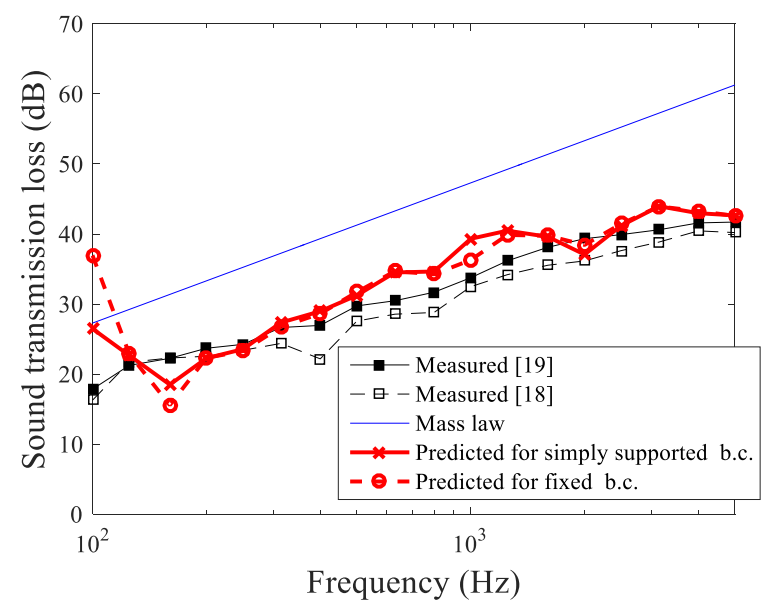

Fig. 30. Comparison of STLs obtained from the numerical simulation and experiments. 
grow as frequency increases. It is sure that STL by the mass law is not suitable for predicting the STL for the complex shaped panel. The WFE/BE results for the simply supported and fixed boundary conditions have similar features with the measured ones in general above 125 $\mathrm{Hz}$ with about 3 5 dB margins. However, the predicted results are about 3 5 dB higher on average than the measured ones between $600 \mathrm{~Hz}$ and $1.25 \mathrm{kHz}$.

\section{Conclusions}

The vibro-acoustic behaviour of a complex shaped extruded panel has been investigated by using the waveguide finite element and boundary element (WFE/BE) method.

In terms of the mechanical excitation, two different types of excitation position were considered; on a strip or on an internal stiffener. It was found that the strip excitation generates localised waves strongly while the stiffener excitation makes global wave modes which are limited to low wavenumbers. Due to this difference in wavenumber domain responses, the strip excitation drops the radiation efficiency at mid frequencies compared to the stiffener excitation because the localized waves existing above the acoustic wavenumber do not radiate sound into far field in spite of their strong vibration. For an acoustic excitation, a diffuse field STL was predicted for the extruded panel. It had its minimum at $160 \mathrm{~Hz}$ and then increased as frequency grows having repeated slight dips at frequencies where the strip mode waves are generated (globally and locally). The STL predicted was not much dependant on the boundary conditions of the panel at mid to high frequencies. The effect of the internal air between the top and bottom plates was not much considerable in terms of the radiation efficiency and STL due to the strong mechanical coupling between the two outer plates by the interior stiffeners. Meanwhile, the applied spatial windows increased the STL at frequencies below $400 \mathrm{~Hz}$.

The predicted results were validated from experiments using a $1.5 \mathrm{~m}$ length extruded panel. It was observed from this comparison that the predicted radiation efficiency for the strip excitation gives better agreement to the measured ones than that for the stiffener excitation. This is because the waves generated by the stiffener excitation have low wavenumbers. Hence, the waveguide assumption in WFE/BE analysis does not suitable to the 1.5 m length panel for the stiffener excitation. In terms of the STL, WFE/BE results agreed with the measured ones within about $3 \mathrm{~dB}$ margins at frequencies above $200 \mathrm{~Hz}$. 
In this study, the WFE/BE method was validated with the results measured from a short length panel of $1.5 \mathrm{~m}$. The measured and predicted results showed fair agreements in general but revealed some discrepancies, especially when the waves of the low wavenumbers are dominant. Nevertheless, it was shown that the WFE/BE analysis will be helpful to study design measures to improve the vibro-acoustic performance of the extruded panel, particularly for high frequencies where the conventional FE/BE method is unsuitable.

\section{Acknowledgements}

The measurement of point mobility was conducted by Yumei Zhang and we are grateful to her for sharing data for the comparison of numerical results with experiments. This research was supported by the Basic Science Research Program through the National Research Foundation of Korea (NRF), funded by the Ministry of Education (2012R1A1A1002779).

\section{References}

[1] G. Xie, D. J. Thompson and C. J. C. Jones, The radiation efficiency of baffled plates and strips, Journal of Sound and Vibration 280, 181-209, 2005.

[2] G. Xie, D. J. Thompson and C. J. C. Jones, A modelling approach for the vibroacoustic behaviour of aluminium extrusions used in railway vehicles, Journal of Sound and Vibration 293, 921-932, 2006.

[3] I. Prasetiyo, Investigation of sound transmission in lightweight structures using a waveguide finite element/boundary element approach, $\mathrm{PhD}$ Thesis, University of Southampton, 2012.

[4] U. Orrenius and S. Finnveden, Calculation of wave propagation in rib-stiffened plate structure, Journal of Sound and Vibration 198(2), 203-224, 1996.

[5] C. M. Nilsson, Waveguide finite elements applied on a car tyre, PhD Thesis, KTH, 2004.

[6] J. Ryue, D. J. Thompson, P. R. White and D. R. Thompson, Investigations of propagating wave types in railway tracks at high frequencies, Journal of Sound and Vibration 315, 157-175, 2008. 
[7] J. Ryue, D. J. Thompson, P. R. White and D. R. Thompson, Decay rates of propagating waves in railway tracks at high frequencies, Journal of Sound and Vibration 320, 955976, 2009.

[8] H. Kim and J. Ryue, Sound radiation from strip plates with longitudinal stiffeners using waveguide finite and boundary element methods, Journal of Mechanical Science and Technology 28(7), 2527-2534, 2014.

[9] Y. Zhang, D. J. Thompson, G. Squicciarini, J. Ryue, X. Xiao and Z. Wen, Sound transmission loss properties of truss core extruded panels, Applied Acoustics 131, 134$153,2018$.

[10] H. Kim and J. Ryue, Numerical analysis on sound transmission loss at high frequencies through extruded panels for railway vehicles, Transactions of the Korean Society for Noise and Vibration Engineering 28(3), 264-270, 2018.

[11] N. J. Shaw, The prediction of railway vehicle internal noise using statistical energy analysis techniques, MSc Thesis, Heriot-Watt University, Edinburgh, 1990.

[12] G. Xie, D. J. Thompson and C. J. C. Jones, Mode count and modal density of structural systems: relationships with boundary conditions, Journal of Sound and Vibration 274, 621-651, 2004.

[13] D. Chronopoulos, M. Ichchou, B. Troclet and O. Bareille, Computing the broadband vibroacoustic response of arbitrarily thick layered panels by a wave finite element approach, Applied Acoustics 77, 89-98, 2014.

[14] T. Kohrs, Wave propagation in light weight plates with truss-like cores, $\mathrm{PhD}$ Thesis, TU Berlin, 2008.

[15] U. Orrenius, H. Liu, A. Wareing, S. Finnveden and V. Cotoni, Wave modelling in predictive vibro-acoustics: Applications to rail vehicles and aircraft, Wave Motion 51, 635-649, 2014.

[16] M. Villot, C. Guigou and L. Gagliardini, Predicting the acoustical radiation of finite size multi-layered structures by applying spatial windowing of infinite structures, Journal of Sound and Vibration 245(3), 433-455, 2001.

[17] J. Legault, A. Mejdi and N. Atalla, Vibro-acoustic response of orthogonally stiffened panels: the effects of finite dimensions, Journal of Sound and Vibration 183, 59285948, 2011.

[18] A. D. Müller, Acoustical investigation of an extruded aluminium railway vehicle floor panel, MSc Thesis, University of Southampton, 2004. 
[19] C. M. Nilsson, A. N. Thite, C. J. C. Jones and D. J. Thompson, Estimation of sound transmission through extruded panels using a coupled waveguide finite elementboundary element method, Proceedings of the 9th International Workshop on Railway Noise, Notes on Numerical Fluid Mechanics and Multidisciplinary Design 99, 306-312, 2007.

[20] C. M. Nilsson, C. J. C. Jones, D. J. Thompson and J. Ryue, A waveguide finite element and boundary element approach to calculating the sound radiated by railway and tram rails, Journal of Sound Vibration 321, 813-836, 2009.

[21] J. Ryue, S. Jang and D. J. Thompson, A wavenumber domain numerical analysis of rail noise including the surface impedance of the ground, Journal of Sound Vibration 432, 173-191, 2018.

[22] C. M. Nilsson, J. Ryue and C. J. C. Jones, Theory manual for WANDS 2.1 Wave number domain FE-BE software for structures and fluids, ISVR Technical Memorandum No. 975, University of Southampton, 2010.

[23] S. Ghinet, N. Atalla and H. Osman, The transmission loss of curved laminates and sandwich composite panels, Journal of the Acoustical Society of America 118(2), 774$790,2005$.

[24] F. Fahy and D. J. Thompson, Fundamentals of sound and vibration, $2^{\text {nd }}$ edition, Boca Raton; CRC Press, 2015.

[25] N. C. Perkins and C.D. Mote Jr., Comments on curve veering in eigenvalue problems, Journal of Sound Vibration 106(3), 451-463, 1986.

[26] J. Brunskog, The influence of finite cavities on the sound insulation of double-plate structures, Journal of the Acoustical Society of America 117(6), 3727-3739, 2005.

[27] H. Kuttruff, Room acoustics, sixth edition, CRC Press, 2016.

[28] F. Fahy and P. Gardonio, Sound and structural vibration: radiation, transmission and response, 2006.

[29] ISO 140-3:1994, Acoustics - Measurement of sound insulation in buildings and of building elements - Part 3: Laboratory measurement of airborne sound insulation of building elements. 